\title{
Travail collectif et usages du numérique : Quelle catégorisation de pratiques chez des enseignants français à l'école et au collège?
}

\section{Collective work and uses of digital technology: What categorization of practices among French teachers at school and college?}

\section{Trabajo colectivo y usos de la tecnología digital: ¿Qué clasificación de las prácticas de los profesores franceses en las escuelas y colegios?}

Luc Massou, maître de conférences

Université de Lorraine, France

luc.massou@univ-lorraine.fr

Jean-Baptiste Lanfranchi, maître de conférences

Université de Paris/Université Gustave Eiffel, France

jean-baptiste.lanfranchi@parisdescartes.fr

Brian Chauvel, ingénieur de recherche

Université de Lorraine, France

brian.chauvel@univ-lorraine.fr

Stéphanie Fleck, maître de conférences

Université de Lorraine, France

stephanie.fleck@univ-lorraine.fr

RÉSUMÉ

Dans le cadre du projet e-TAC Environnements Tangibles et Augmentés pour l'Apprentissage Collaboratif, une enquête par questionnaire a été menée en ligne auprès d'enseignants de cycles 3 et 4 de Moselle (France) pour mieux décrire leurs pratiques de travail collectif entre pairs et en classe, avec ou sans numérique. Au travers de l'analyse des 972 réponses 
obtenues, nous traiterons deux questions principales : quelles sont les catégories de pratiques déclarées par les enseignants et la place qu'y occupent les outils et ressources numériques? De quoi dépend la fréquence du travail en groupe de leurs élèves à l'école et au collège? Nos traitements statistiques sur les données relatives aux pratiques des professeurs des écoles et des enseignants de collèges permettent, d'une part, d'identifier quatre catégories de variables associées aux pratiques de travail collectif chez les enseignants, qui s'agrègent selon deux axes paradigmatiques (enseignant/apprenant, autocentré/hétérocentré) et, d'autre part, d'étudier la force de prédiction de six catégories de variables du travail en groupe avec leurs élèves, où l'impact des usages d'outils et ressources numériques s'avère finalement très secondaire.

Mots-clés : travail collectif, enseignants, outils et ressources numériques, école, collège, prédicteurs

\section{ABSTRACT}

As part of the e-TAC project Tangible and Augmented Environments for Collaborative Learning, a questionnaire survey was conducted online among teachers of cycles 3 and 4 in Moselle (France) to better describe their collective work practices peers and in class, with or without digital technology. By analyzing the 972 responses obtained, we address two main questions: What are the categories of practices declared by teachers and the place of digital tools and resources in them? On what depends on the frequency of group work by their students at school and college? Our statistical processing of data on school and college teachers' practices allowed us to identify four categories of variables associated with the groups' practices. Results show they are aggregated along two paradigmatic axes (teacher/learner, self-centred/heterocentric). On the other hand, the analysis of the predictive power of six categories of group work variables shows that the impact of the use of digital tools and resources ultimately proves to be very secondary.

Keywords: group work, teachers, digital tools and resources, school, college, predictors

\section{RESUMEN}

En el marco del proyecto e-TAC "Entornos Tangibles y Aumentados para el Aprendizaje Colaborativo", se realizó una encuesta por cuestionario en línea entre los profesores de los ciclos 3 y 4 en Moselle (Francia) para describir mejor sus prácticas de trabajo colectivo entre pares y en clase, con o sin medios digitales. A través del análisis de las 972 respuestas obtenidas, abordaremos dos preguntas principales: ¿cuáles son las categorías de prácticas declaradas por los profesores y el lugar que ocupan las herramientas y recursos digitales en ellas? ¿De qué depende la frecuencia del trabajo en grupo de sus estudiantes en la escuela y en la universidad? Nuestro procesamiento estadístico de los datos sobre las prácticas de los profesores de escuelas y universidades nos permite, por un lado, identificar quatro categorías de variables asociadas a las prácticas de trabajo en grupo de los profesores, que se agregan a lo largo de dos ejes paradigmáticos (profesor/aprendiz, autocentrado/ heterocéntrico), y, por otro lado, estudiar el poder predictivo de seiz categorías de variables de trabajo en grupo con sus alumnos, donde el impacto del uso de herramientas y recursos digitales resulta ser muy secundario.

Palabras clave: trabajo en grupo, profesores, herramientas y recursos digitales, escuela, universidad, pronosticadores 


\section{Introduction}

Le point de départ de cette recherche s'inscrit dans le cadre du projet de recherche collective e-TAC Environnements Tangibles et Augmentés pour I'Apprentissage Collaboratif (2017-2021)'. L'objectif principal de ce projet est de concevoir des interfaces de nouvelle génération, tangibles et transparentes (sans clavier ni souris) soutenant l'apprentissage collaboratif en contexte scolaire (pour un exemple d'interface, voir Giraudeau et al., 2019). Selon Dillenbourg (1999), l'apprentissage collaboratif est une situation dans laquelle deux personnes ou plus apprennent, ou tentent d'apprendre, quelque chose ensemble de manière interactive, synchrone et négociable. Selon Conein (2004), l'apprentissage collaboratif, conséquence d'une action collective (par exemple: travail en groupe avec ou sans coopération ou collaboration) est optimal lorsque la dynamique de cette action tire parti d'un contexte à la fois organisationnel et technologique, où les groupes comme les artefacts agissent comme des supports externes à l'augmentation de la connaissance. Celui qui apprend est donc ici tout aussi influencé par l'environnement physique (le matériel numérique ou non à sa disposition...) que par l'environnement social (rôle et place de l'enseignant...) dans et avec lesquels il interagit.

Fondé sur les principes de la conception centrée sur l'utilisateur (voir par exemple : Norman et Draper, 1986; Oviatt, 2006), ce projet cherche à répondre au mieux aux besoins rencontrés lors d'activités collectives en vue de soutenir, voire améliorer l'apprentissage des élèves et les pratiques enseignantes associées. Pour Oviatt (2006), l'approche centrée sur l'utilisateur préconise une approche plus prometteuse et durable qui est, dans un premier temps, de copier le comportement naturel des utilisateurs en incluant toutes les contraintes liées à leurs aptitudes à participer, à apprendre et à exécuter. Selon lui, les interfaces peuvent ainsi être conçues pour être plus intuitives, plus faciles à apprendre et exemptes d'erreurs d'utilisation. De plus, identifier et prendre en compte les besoins et pratiques d'un point de vue pragmatique, mais aussi du point de vue des valeurs et normes structurant les choix des enseignants, facilite l'acceptabilité et donc l'usage des outils numériques au cours de situations d'apprentissage en contexte scolaire (Tondeur et al., 2008). Mais en contexte éducatif, si la démarche de conception centrée sur l'utilisateur diffère peu de celle définie habituellement (voir Loup-Escande et al., 2015), au travers de la réalisation de quatre tâches selon une approche souvent itérative (de type exploration, idéation/spécification, prototypage et évaluation), la toute première tâche réclame une attention particulière. En effet, il s'agit de comprendre et de caractériser le contexte d'utilisation, les besoins et représentations ${ }^{2}$ des utilisateurs pour les activités ciblées par l'interface en procédant à une analyse de l'environnement dans lequel l'outil sera intégré. Cette étape permet également de mieux connaître la place actuellement occupée par les usages d'outils et ressources numériques chez les acteurs visés, pour ne pas en ignorer la généalogie (Jouët, 2000) et ainsi concevoir de futures interfaces en toute connaissance de cause. Cerner ces aspects nécessite donc des approches de recherche exploratoires (Trudel et al., 2007) dans un but à la fois descriptif et inductif. En s'appuyant sur des observations passives assistées par la vidéo en contexte de classe, des entretiens et des enquêtes, le projet e-TAC nourrit ainsi tout le processus de conception de ses environnements de connaissances issues de recherches permettant de baliser une certaine réalité scolaire : pratiques effectives, mais aussi représentations des enseignants sur leurs pratiques.

\footnotetext{
${ }^{1}$ Site Web du projet : http://e-tac.univ-lorraine.fr/ (consulté le 10/06/2020).

${ }^{2}$ Nous entendons ici les représentations comme des systèmes de connaissances qu'un sujet mobilise spontanément face à une question ou à un problème, que ceux-ci aient ou non fait l'objet d'un apprentissage [Reuter et al., 2010].
} 
Les résultats de l'étude que nous présentons ici s'inscrivent donc dans ce cadre et répondent à deux principaux objectifs: faire l'état des lieux des pratiques enseignantes actuelles concernant le travail collectif entre pairs et en classe, et identifier des déterminants pouvant avoir un impact sur leurs dynamiques collectives en contexte scolaire. Elle vise ainsi à recenser, qualifier, voire expliquer ce qui sous-tend la mise en œuvre de ces pratiques entre pairs et en classe, et d'y identifier la place éventuelle des outils et ressources numériques ${ }^{3}$. Dans ce but, une enquête par questionnaire en ligne auprès des écoles primaires et collèges de Moselle (France) a été menée. Elle interroge plus spécifiquement les enseignants des cycles 3 et 44 , niveaux ciblés par le projet e-TAC $(n=972)$. Pour cet article, nous contextualiserons d'abord notre étude par rapport aux enjeux socioprofessionnels du travail collectif chez les enseignants et aux manques identifiés dans ses définitions actuelles, pour ensuite expliciter nos choix méthodologiques, présenter les résultats détaillés obtenus et les mettre en discussion par rapport à l'état de l'art sur ces thématiques.

\section{Éléments de contexte}

\section{Enjeux sociétaux, institutionnels et professionnels du travail collectif}

Tout d'abord, il est à noter que le travail actuel des enseignants s'inscrit dans un contexte socioprofessionnel et institutionnel où la demande de travailler et de faire travailler collectivement est forte. Comme le souligne Gibert (2018, p. 2), les injonctions à penser collectif sont largement présentes dans le référentiel des compétences des métiers du professorat et de l'éducation mis en place en France à l'occasion de la loi sur la refondation de l'école en 2013 : compétences sur les valeurs partagées et les principes fondamentaux, référence à la coopération (entre élèves, en équipe, avec les parents, les partenaires), nécessaire contribution à la communauté éducative et engagement dans une démarche individuelle et collective de développement professionnel. Selon Lessard et al. (2009), les arguments justifiant ces injonctions à collaborer ou au travail collectif sont triples :

- $\quad$ passer de la résolution collective de problèmes à la cogestion démocratique;

- apprendre de l'organisation (modes partagés d'analyse, compréhension et préparation des situations);

- professionnaliser (communauté d'apprentissage et augmentation de l'efficacité des enseignants).

Ce contexte a des implications sur les activités professionnelles des enseignants, qu'elles soient auprès des élèves ou au sein des équipes pédagogiques. Marcel et al. $(2007$, p. 8$)$ faisaient déjà ce constat de l'évolution de la figure traditionnelle du métier d'enseignant vers une figure composite d'un professionnel exerçant dans un établissement scolaire confronté à une recrudescence de partenaires diversifiés et travaillant de plus en plus fréquemment avec les autres enseignants hors de la présence des élèves et parfois, mais plus rarement, en leur présence. Cette évolution semble justifiée par les apports constatés sur le développement professionnel collectif et continu des enseignants par différentes études sur le travail collectif, avec des impacts sur trois niveaux (Gibert, 2018; Baudrit, 2007) :

- les enseignants eux-mêmes : confiance en soi, sentiment d'efficacité personnelle, motivation, engagement, stratégies d'enseignement et d'apprentissage pour s'adapter aux besoins des élèves, innovation pédagogique;

\footnotetext{
${ }^{3}$ Par outils et ressources numériques, nous entendons à la fois les logiciels, supports, environnements de travail et applications informatiques (en ligne et hors ligne, mobiles ou fixes), ainsi que les contenus numériques (documentation, manuels, sites Web, publications...) utilisés par les enseignants et les élèves.

${ }^{4}$ Actuellement en France, le cycle 3 couvre les deux dernières années de l'école primaire et la première année du collège, et le cycle 4 couvre les trois dernières années du collège. Ces cycles concernent donc des élèves de 9 à 12 ans (cycle 3 ) et de 12 à 15 ans (cycle 4 ) en moyenne.
} 
- les élèves et leurs apprentissages : améliorations dans le domaine affectif, social et cognitif, développement éthique;

- l'organisation : climat innovant, meilleure adaptation, équité, attention aux besoins des élèves, structure moins hiérarchique, culture professionnelle de recherche, culture d'établissement.

Cependant, comme l'affirme Cristol (2016), il ne suffit pas de mettre des moyens à la disposition de groupes pour qu'ils se transforment en communautés d'apprentissage. Aussi, face à ces évolutions et à ces enjeux, il semble important d'identifier quels sont les contours actuels du travail collectif en contexte scolaire afin de contribuer à catégoriser des pratiques enseignantes dont la définition est encore plurielle.

\section{Une définition des pratiques qui reste encore floue}

Malgré ce contexte sociétal et institutionnel favorable à l'évolution du travail collectif chez les enseignants, Gibert (2018) a fait récemment le constat que sa définition reste encore floue, entre informel et institué : approche par continuum, différences conceptuelles (échanges informels, travail en petits groupes choisis, groupes de travail plus formels, communautés d'apprentissage ou de pratique). Le travail collectif peut ainsi aller de la simple agrégation d'individus indépendants jusqu'à un fort travail d'équipe en interdépendance, de la collaboration mutuelle et coordonnée (pour une résolution de tâche visant une efficacité professionnelle) à la coopération (comme réalisation d'un travail partagé par des sujets autonomes et volontaires). Différentes études appuient ce caractère multiple du concept. Pour ce qui est du travail collectif entre pairs, un état de l'art mené à partir d'une métarelecture de 82 études scientifiques publiées après l'année 2000 sur le sujet (Vangrieken et al., 2015) a ainsi identifié six manières différentes de le nommer :

- $\quad$ Teacher collaboration $(n=13)$;

- Professional (learning) communities or PLC (6) : un groupe professionnel, partageant un travail commun et certaines valeurs, normes ou conceptions;

- Communities (of practice) or CoP (5) : un bloc constitutif des PLC, incluant entraide mutuelle, collaboration et collégialité;

- Teacher teams (52) : réfère davantage à l'équipe pédagogique complète et moins au fait qu'ils collaborent dans une équipe ou pas;

- Teacher (learning) groups or Departements (3): collection d'individus partageant la même catégorisation et identité sociales;

- $\quad$ Other (3).

Pour sa part, Dupriez (2010) affirme que les pratiques collectives des enseignants sont influencées par des conceptions différentes des pratiques éducatives, influences qu'il regroupe selon trois dimensions en fonction de la manière dont peuvent être perçues les finalités éducatives poursuivies, les notions de savoir, de formes ou de modalités d'apprentissage :

- mission d'instruction vs mission d'éducation, qui nécessite un travail plus intégré et qui ne peut reposer sur l'addition simple d'un certain nombre de savoirs;

- apprentissages décontextualisés vs contextualisés, plus favorables à des pratiques telles que la pédagogie du projet, les approches interdisciplinaires et l'enseignement des compétences, pour 
préparer les élèves à faire usage de leurs connaissances pour comprendre et agir dans leur environnement;

- pratiques pédagogiques convergentes vs divergentes, associées à des dispositifs pédagogiques tels que la pédagogie du projet, l'apprentissage par résolution de problèmes ou encore tout dispositif laissant une large place au conflit sociocognitif, qui créent inévitablement une plus grande incertitude sur la conduite des situations d'enseignement.

Dupriez émet l'hypothèse que plus un système scolaire définit ses objectifs en termes d'éducation, valorise l'appropriation de savoirs contextualisés et développe des dispositifs pédagogiques laissant de la place aux apports des élèves, plus ce système s'appuiera sur du travail collectif et de la collaboration entre enseignants. Au-delà de ces conceptions et facteurs d'influence multifactoriels du travail collectif, l'auteur parle aussi de récit mythique. Selon son étude menée en 2010, la distance demeurait alors encore grande, dans le monde francophone en tout cas, entre l'injonction au projet et au travail en équipe (collectif), et les pratiques effectives au sein des écoles. Le travail collectif serait comme une histoire qu'on nous raconte, qui commence à s'inscrire dans l'univers scolaire, porteuse d'un projet symbolique, mais qui n'est pas complètement vraisemblable, car difficile à observer dans l'activité quotidienne des enseignants. Or, qu'en est-il de la réalité des pratiques actuelles, près de dix ans après?

En écho, il n'est pas simple non plus de cerner la place du numérique dans ces pratiques collectives enseignantes. Si l'on se réfère à la seule enquête nationale Profetic ${ }^{5}$ disponible en France sur les usages pédagogiques du numérique, et qui fait figure de référence, car menée depuis 2011 par le ministère de l'Éducation nationale et de la Jeunesse auprès de 5000 enseignants de premier et second degrés, les liens entre travail collectif entre pairs et usages des outils numériques y occupent une place toute relative. Dans leurs dernières éditions 2017 (sur le premier degré, $n=1387$ ) et 2018 (sur le second degré, $n=2633$ ), et sur la question spécifique du travail collectif entre pairs, le numérique y est perçu comme un avantage pour travailler et partager avec des collègues pour respectivement $72 \%$ et $85 \%$ des répondants des premier et second degrés, bien loin de la diversification des pratiques pédagogiques (93\% et $91 \%$ ) et de la réalisation des préparations de séquences (92\% et $95 \%)$. Mais de manière plus générale, le recours à la manipulation de matériels numériques en classe par les élèves eux-mêmes reste encore faible (selon respectivement $25 \%$ et $57 \%$ des enseignants des premier et second degrés), les usages restant concentrés sur la mise en œuvre de séquences pédagogiques en classe sans numérique (77\% et $84 \%$ ). Ces enquêtes ne précisent cependant pas si les usages diffèrent lors de travaux collectifs avec leurs pairs et leurs élèves.

À la lumière de ce contexte arborant des visions multiples et constatant le manque d'études descriptives récentes, il semble donc pertinent d'interroger trois niveaux d'influence potentiels sur ces pratiques : le travail collectif entre pairs, le contexte institutionnel, mais aussi matériel (dont le numérique fait partie) dans lequel il s'inscrit, et les pratiques pédagogiques qu'y s'y rapportent, en prenant comme point d'entrée la perception des enseignants sur ces trois dimensions : quelles sont les formes de travail collectif les plus souvent mobilisées par les enseignants? Quelles dimensions dominent parmi la diversité des représentations proposées? Le matériel que l'on pourrait qualifier d'habituel en classe (numérique ou non) y trouve-t-il une place particulière et, si oui, laquelle? C'est à ces questions que notre enquête a tenté de répondre afin de pouvoir dégager une proposition de catégorisation de ces pratiques de travail collectif entre pairs et au sein de leur classe, et en évaluant la place qu'y occupent - ou non - les outils et ressources numériques mis à leur disposition dans leur environnement de travail quotidien. Les usages du numérique évoqués dans le titre de notre article ne s'entendent donc pas de manière générale, mais se rapportent uniquement à leur place et impact éventuels au sein de ces pratiques de travail collectif (cf. : volets « $\mathrm{C}$ » et « $\mathrm{e}$ » du questionnaire présenté ci-après) afin de pouvoir in fine les prendre en compte pour

\footnotetext{
${ }^{5}$ Accès : https://eduscol.education.fr/cid60867/l-enquete-profetic.html (consulté le 15 mai 2020).
} 
la conception d'interfaces numériques de nouvelle génération, comme expliqué en introduction. Le taux de réponse important obtenu $(n=972)$ nous permettra également d'apporter des éléments complémentaires aux typologies et aux travaux précités.

\section{Méthodologie et terrain de recherche}

Les objectifs de cette action de recherche étaient donc doubles: connaître les manières de travailler collectivement en contexte scolaire et identifier des catégories de pratiques de ce travail collectif entre pairs et en classe selon différentes variables (fréquence, types d'activités, modalités, ressources utilisées, usage du numérique, taille des groupes...). Pour cela, la méthode retenue a été l'enquête par questionnaire anonyme diffusée à l'ensemble des écoles et collèges de la Moselle et à destination des enseignants de cycles 3 et 4 uniquement. En fonction du taux de retour obtenu, cette approche quantitative permet le recueil d'un grand nombre d'informations factuelles dans un but descriptif sur les pratiques déclarées par les enseignants d'un territoire donné. Pour atteindre une visée exploratoire, elle fait également le choix d'un parti-pris empirique fondé sur l'importance accordée à la collecte de données préalables et à la manière dont les acteurs concernés s'expriment, à partir de quoi nous pourrons dégager une catégorisation des pratiques déclarées. La limitation de la diffusion à un territoire départemental était à la fois une attente de l'appel à projet e-FRAN Des territoires éducatifs d'innovation numérique, dont dépendait le financement de notre projet lauréat eTAC ${ }^{6}$, et permettait de garantir une certaine cohérence du contexte institutionnel et éducatif dans les choix politiques régionaux de l'Académie (Nancy-Metz, dans notre cas). Enfin, dans une logique davantage qualitative et compréhensive, cette enquête a également été complétée par 21 entretiens semi-directifs de professeurs des écoles et de collège, mais ils ne feront pas l'objet de notre analyse pour cet article.

\section{Dimensions interrogées et modalités de construction de l'enquête}

Le questionnaire est une enquête en ligne diffusée via l'outil LimeSurvey. II s'articule en cinq volets différents listés ci-dessous, comprenant chacun plusieurs questions, les volets « $a$ » et « $d$ » se rapportant à la nature du travail collectif et aux activités pédagogiques, les volets $\| \mathrm{b}$ », 《 $\mathrm{c}$ » et « $\mathrm{e}$ " à l'environnement de travail incluant le numérique :

a. Le travail collectif entre enseignants

b. Le contexte professionnel du travail entre enseignants

c. L'usage du numérique par les enseignants

d. Le travail collectif des élèves

e. Les outils de la classe

L'ensemble représente 62 questions fermées avec un total de 240 items ayant de deux à cinq modalités de réponse chacun (par exemple: "Oui/Non» $=2$ modalités, "Pas du tout d'accord/Pas d'accord/Indifférent/Plutôt d'accord/Tout à fait d'accord » $=5$ modalités). Un sixième volet de 19 questions interroge les enseignants sur des dimensions sociodémographiques (établissement, bassin, statut actuel, type et nombre de classes en charge et dans quels cycles, formations initiale et continue suivies, discipline, âge, ancienneté...) en vue de préciser les attributs des répondants.

\footnotetext{
${ }^{6}$ Voir présentation de l'appel et liste des 22 projets lauréats en 2017 : https://www.gouvernement.fr/efran-les-22-laureats (consulté le 01/06/2020).
} 


\section{CHOIX DE CONCEPTION DU QUESTIONNAIRE}

Comme nous l'expliquons en introduction et dans la précédente section, l'enquête vise à interroger trois principales dimensions selon une visée descriptive et exploratoire : le travail collectif entre pairs et en classe, les pratiques pédagogiques et l'environnement institutionnel et matériel afférents. Le choix des questions a donc été pensé afin de faire émerger une photographie la plus détaillée possible à un instant t et sur un territoire académique donné, des modalités et de la nature des pratiques enseignantes de travail collectif entre pairs et dans leur classe. Pour cela, et en prenant appui sur la connaissance du terrain et les retours issus d'un premier prétest auprès des partenaires académiques du projet e-TAC (enseignants, formateurs d'enseignants, inspecteur), le questionnaire a été pensé pour couvrir le champ de réponses le plus complet possible parmi lesquelles figuraient notamment les dimensions suivantes : la fréquence des activités collectives entre collègues, le type d'activités collectives, l'usage de ressources et outils numériques, la répartition des tâches entre pairs et entre élèves, les compétences du socle visées en termes d'apprentissage, les modalités d'évaluation. Pour évaluer et ajuster à la fois la longueur du questionnaire et la bonne formulation des différentes questions, un second prétest a été réalisé auprès de dix enseignants de cycles 3 et 4 , partenaires du projet e-TAC.

Les questions ont été formulées dans un langage générique, évitant le vocabulaire trop spécialisé pour limiter les biais méthodologiques de formulation. Concernant le choix des dimensions en lien avec les apprentissages des élèves visés par le travail collectif mis en place par les enseignants, le questionnaire a repris les attendus officiels tels qu'ils sont formulés dans le socle commun de connaissances, de compétences et de culture en fin de cycles 3 et $4^{7}$ (dicté par le ministère de l'Éducation nationale et de la Jeunesse en France en 2015), seul référentiel commun aux deux degrés et à toutes les disciplines enseignées par les enseignants interrogés.

\section{MODALITÉS DE DIFFUSION}

Sur le plan éthique, seul l'usage des adresses académiques des établissements visés (et non celles de leurs enseignants afin de garantir leur anonymat) listées dans l'annuaire de l'éducation a été retenu pour diffuser le questionnaire et pour gérer les relances collectives. Au préalable, le directeur académique des services de l'Éducation nationale (DASEN) a été contacté pour accord et pour qu'il en informe les chefs d'établissement. Le lien d'accès au questionnaire leur a ensuite été envoyé par mél. mi-février 2018, suivi de quatre relances entre fin février et début mai 2018. Pour ce faire, les messages d'invitation à répondre au questionnaire et les relances ont été adressées exclusivement aux proviseurs des 104 collèges et aux directeurs des 652 écoles primaires de Moselle afin qu'ils les relaient par mél. à leurs collègues enseignants en cycles 3 et 4 uniquement. L'envoi par la voie hiérarchique avait pour objectif de garantir une diffusion efficace de l'enquête et espérer un bon taux de complétude. Bien qu'ayant transité par cette voie interne à l'institution, cette diffusion en ligne, anonyme et rendue neutre vis-à-vis de l'enquêteur, vise à limiter les biais de désirabilité sociale (Butori et Parguel, 2010).

Au cours de l'année scolaire 2017-2018, année de diffusion de l'enquête, le territoire du département de Moselle comptait un total de 4801 enseignants des premier et second degrés ${ }^{8}$. Le questionnaire cible plus particulièrement la population des 1230 enseignants (dont $56,7 \%$ sont des femmes) exerçant en collège et des $919^{\circ}$ enseignants (dont $81,2 \%$ sont des femmes) exerçant à l'école primaire.

\footnotetext{
${ }^{7}$ Socles communs de connaissances, de compétences et de culture en fin de cycle 3 (https://cache.media.eduscol.education.fr/file/College 2016/74/4/RAE Evaluation_socle cycle 3 643744.pdf, consulté le 10/06/2020) et de cycle 4 (https://cache.media.eduscol.education.fr/file/College 2016/74/6/RAE Evaluation socle cycle 4 643746.pdf, consulté le 10/06/2020). Les programmes scolaires officiels prennent appui sur ces socles.

${ }^{8}$ Source : site Open Data du ministère de l'Éducation nationale et de la jeunesse. Accès : https://data.education.gouv.fr/explore/dataset/fr-eneffectifs-des-personnels-des-ecoles-et-etablissements-du-2nd-degre/export/?refine.annee scolaire=2018-2019\&refine.academie=NANCYMETZ\&refine.code departement=057 (consulté le 10/06/2020).
} 


\section{Les traitements des données collectées}

Dans un premier temps, les questions à choix multiples ayant $n$ énoncés (ex. : Q.25 "Quels types d'évaluation utilisez-vous pour les travaux de groupe? " proposait six choix possibles), représentant $n$ variables à deux modalités (" Oui/Non »), ont été regroupées d'une manière plus sensible à leurs affinités internes (coprésences dans les réponses, association par les répondants) et distinctions mutuelles (coabsence) pour limiter les biais statistiques. De la même façon, les questions identiques interrogeant dans un cas les travaux en groupe, dans l'autre les travaux avec le numérique (ex.: Q.24 « Je pense que [le travail de groupe] (ou Q.35 [travailler avec le numérique]) permet à mes élèves de... ») ont été regroupées en une variable unique à deux modalités. L'analyse a ainsi été appliquée à un total final de 120 variables.

\section{ANALYSE DESCRIPTIVE}

Un traitement statistique par tris à plat a d'abord été appliqué pour connaître les résultats bruts de l'échantillon étudié, tous niveaux confondus. Cela vise à caractériser la population et à identifier les proportions relatives de réponses positives obtenues pour chacune des 120 variables, sur lesquelles a été ensuite appliquée la classification ascendante hiérarchique pour soutenir l'analyse des résultats obtenus. Ce traitement s'est effectué via le logiciel SPSS pour chacune des questions posées. Une analyse de la variance (Anova) a été également effectuée en vue d'identifier d'éventuelles différences significatives entre les enseignants des premier et second degrés.

\section{CLASSIFICATION ASCENDANTE HIÉRARCHIQUE}

En vue d'identifier ce qui caractérise les enseignants-répondants qui collaborent entre eux, qui font collaborer leurs élèves et la place du numérique dans ces pratiques, une classification ascendante hiérarchique $(\mathrm{CAH})$ a été appliquée sur l'ensemble des variables. L'objectif est de mettre en évidence les liens de proximités statistiques entre un grand nombre de variables, ici catégorielles, et de faire ressortir les variables expliquant la majorité des réponses. Cette approche par CAH permet ainsi l'exploration de données multivariées. Cela aboutit à la production d'une structure arborescente permettant la mise en évidence de liens hiérarchiques entre variables et la détection d'un nombre de classes de regroupements, ou « clusters », statistiquement similaires dans un grand jeu de données.

Pour construire cette analyse transversale des représentations et pratiques des répondants concernés, un panel cible de 780 enseignants-répondants, appelé échantillon cible, a été retenu parmi l'ensemble des répondants au questionnaire. Cet échantillon cible a pour dénominateur commun d'avoir des réponses positives à quatre questions du questionnaire :

1) Depuis la rentrée, j'ai eu l'occasion de faire un travail collectif avec mes collègues.

2) J'utilise le numérique pour travailler avec mes collègues.

3) Depuis la rentrée, je fais travailler mes élèves en groupe.

4) J'utilise le numérique en classe lors des travaux de groupe.

\footnotetext{
${ }^{9}$ Estimation à partir des données totales, en considérant qu'un tiers des professeurs des écoles (premier degré) exercent potentiellement en
} cycle 3 . 
La classification hiérarchique sur composante principale aboutissant à la $\mathrm{CAH}$ a été effectuée via l'utilisation des packages R FactoMineR (Lê, Josse, et Husson, 2008) et CLustOfVar (Vigneau, Chen et Qannari, 2015).

\section{RÉGRESSION PARTIAL LEAST SQUARE}

Enfin, à partir des variables de l'enquête, deux régressions des moindres carrés partiels (dite régression PLS pour Partial Least Square) ont été réalisées sur 931 répondants, incluant également ceux qui n'ont pas répondu de manière systématique aux quatre questions centrales de l'échantillon cible précédent. L'objectif de ce troisième traitement des données était d'étudier la force de prédiction de six catégories de variables du questionnaire sur la fréquence d'usage du travail en groupe avec leurs élèves : les aspects sociodémographiques et professionnels, l'attitude sur les technologies du numérique et leurs usages, les types de collaboration avec les collègues, les types d'activités de travail en groupe, l'instrumentalité perçue du travail en groupe pour favoriser l'émergence de compétences et les ressources matérielles et numériques mises à la disposition des enseignants. La première régression PLS porte sur les données relatives aux pratiques des professeurs des écoles ( $n=397,66$ variables), la seconde sur des enseignants de collège (sans les enseignants d'éducation physique et sportive ${ }^{10}, n=534,68$ variables).

Nous avons choisi cette méthode statistique du fait de sa grande souplesse pour intégrer de nombreuses variables binaires, ordinales ou d'intervalle, même fortement corrélées entre elles, dont on souhaite voir l'expression d'une manière moins compétitive que la régression multiple usuelle (Tenenhaus, 1998) : l'approche est donc principalement exploratoire. Dans une première étape, la régression PLS regroupe ces variables sous des axes factoriels les plus prédicteurs du critère choisi, pour ensuite calculer des coefficients de régression à partir de cette contribution de chaque variable aux axes factoriels retenus (un seul axe factoriel dans notre cas est pertinent). Les variables peu participatives ont été supprimées de manière itérative des analyses jusqu'à n'avoir plus qu'un lot de variables significativement reliées au critère de fréquence du travail en groupe. L'ensemble des analyses a été réalisé sous le logiciel $R$ version 3.5.3 avec le package PLS (Mevik, Wehrens et Hoyde Liland, 2019). Ces variables prédictrices étaient issues de six catégories (voir tableau 1).

\section{Tableau 1}

Liste des six catégories de variables prédictrices pour la régression PLS

\begin{tabular}{|l|l|}
\hline \multicolumn{1}{|c|}{ Catégorie } & \multicolumn{1}{|c|}{ Variables } \\
\hline Les aspects sociodémographiques et professionnels (C1) & $\begin{array}{l}13 \text { variables pour } \\
\text { l'école, 15 pour le } \\
\text { collège }\end{array}$ \\
\hline L'attitude sur les technologies du numérique et ses usages (C2) & 7 variables \\
\hline Les types de collaboration avec les collègues (C3) & 3 variables \\
\hline Les types d'activités de travail en groupe (C4) & 10 variables \\
\hline L'instrumentalité perçue du travail en groupe pour favoriser l'émergence de compétences (C5) & 15 variables \\
\hline Les ressources matérielles et numériques mises à la disposition des enseignants (C6) & 18 variables \\
\hline
\end{tabular}

\footnotetext{
${ }^{10}$ Ces enseignants ont été retirés du traitement PLS, car leurs réponses présentaient une corrélation assez « mécanique » avec le travail en groupe $(r=.26)$ et beaucoup de questions ne les concernaient pas vraiment (comme l'usage des ressources et outils numériques).
} 


\section{Résultats}

\section{Profil des répondants et de l'échantillon cible}

Pour l'ensemble de l'enquête, 972 questionnaires ont été retournés complets et exploitables, soit $47 \%$ des enseignants du département intervenant en cycles 3 et 4 (selon les données ministérielles disponibles en Open Data : voir note de bas de page 3). Parmi cette population, 575 sont enseignants de collège et 397 en école primaire. La population totale des répondants est très féminisée, tout comme la population du département, en particulier à l'école primaire (voir figure 1). II faut cependant noter que la proportion d'hommes exerçant en collège ayant répondu au questionnaire est bien inférieure encore $(-17,9 \%)$, par rapport à la proportion d'hommes enseignant en cycles 3 et 4 dans le département de la Moselle. Parmi ces répondants, $98,6 \%$ déclarent avoir fait travailler leurs élèves en groupe et $83,8 \%$ avoir travaillé directement avec d'autres enseignants au cours de l'année.

\section{Figure 1}

Proportions relatives de l'échantillon cible pour les $1^{\text {er }}$ et $2^{e}$ degrés

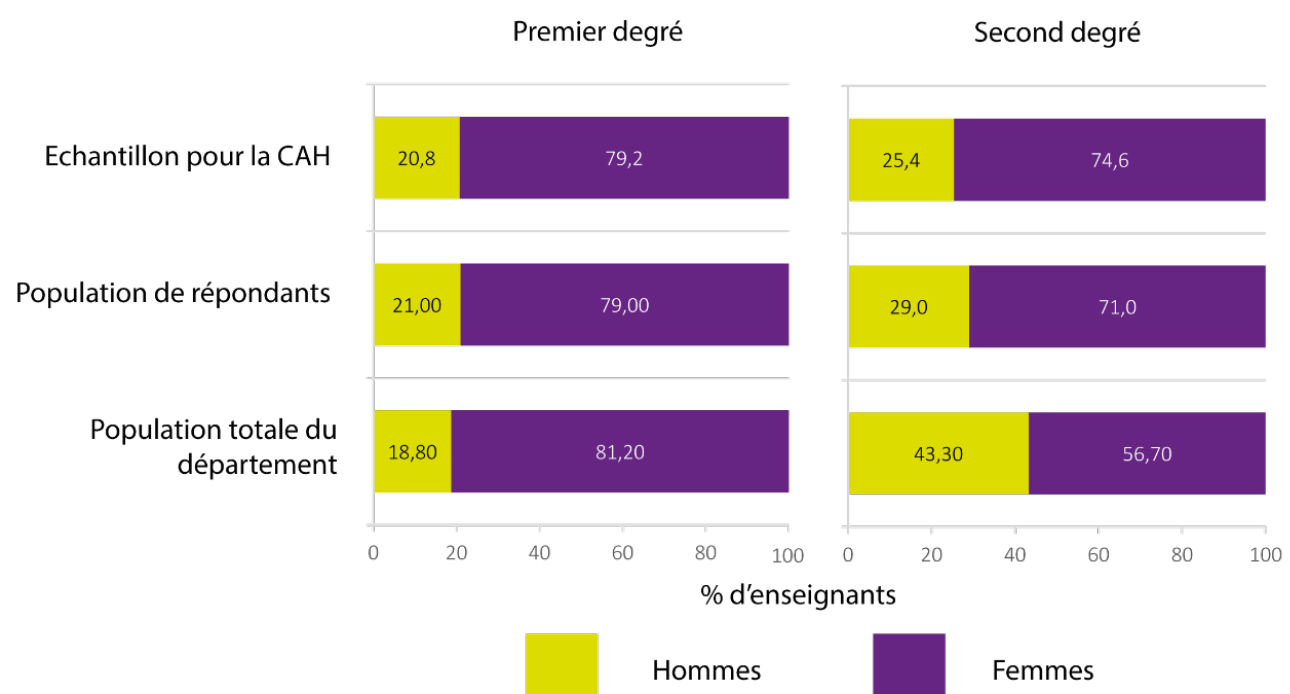

Concernant les 780 répondants de l'échantillon cible retenu pour la $\mathrm{CAH}$ (qui ont répondu positivement aux quatre questions centrales citées supra à la section "Classification ascendante hiérarchique "), ils représentent $80,2 \%$ de la population totale des répondants et lui sont donc très similaires, à l'exception d'un taux de féminisation légèrement plus élevé au collège (voir figure 1). Les quatre bassins du territoire de la Moselle (Metz, Sarre, Saint-Avold et Thionville) y sont représentés à parts quasi égales. Parmi les autres résultats significatifs de l'échantillon, nous noterons que :

- $60 \%$ des répondants de l'échantillon exercent en collège $(n=468)$ contre $40 \%$ en école primaire $(\mathrm{n}=312)$;

- $\quad 19,2 \%$ des répondants sont en réseau d'éducation prioritaire, mais seuls 3,5\% sont enseignants spécialisés;

- $\quad 69 \%$ ont 3 ans et plus années d'ancienneté et les répondants sont presque tous titulaires de leur poste $(92,1 \%)$

- seuls 7,8 \% ont moins de trente ans, les 30-39 et 40-49 ans représentent chacun un tiers du panel et les plus de 50 ans en représentent $20,6 \%$. 


\section{Analyse descriptive des pratiques déclarées de travail collectif}

Les tris à plat permettent une première mise en respective des répondants par variables du travail collectif en distinguant notamment des dimensions centrées sur l'enseignant et sur les élèves. Nous en présentons ici une sélection de réponses significatives.

\section{LE TRAVAIL COLLECTIF CENTRÉ SUR L'ENSEIGNANT : LA PRÉPARATION DES ENSEIGNEMENTS OU DES PROJETS}

Le travail entre collègues est essentiellement consacré à la préparation de leurs enseignements $(55,5 \%)$ ou de projets (65,4\%). Il est intéressant de noter que, dans seuls $13,3 \%$ des cas, ces enseignements sont mis en œuvre pour une classe commune. Le travail de conception des évaluations reste, quant à lui, un travail majoritairement individuel $(76,9 \%)$. Le travail de conception en collectif s'effectue pour le plus grand nombre selon une fréquence mensuelle $(40,3 \%$, contre $22,4 \%$ une fois par semaine, $24,1 \%$ une fois par trimestre et seulement $2,4 \%$ de manière quotidienne), en binôme ou trinôme $(67,6 \%)$ ou en équipe restreinte $(25,5 \%)$ de collègues du même établissement qu'eux $(90,6 \%$ et, dans $43,8 \%$ des cas, ce sont systématiquement les mêmes collègues). Ils consacrent généralement de 30 à 90 minutes $(63,7 \%)$ à ce travail et, dans $94 \%$ des cas, ce travail s'effectue au sein de l'établissement, au domicile d'un enseignant de l'équipe $(41,4 \%)$ et à distance, en particulier pour les enseignants du collège. En effet, l'usage du Web pour travailler en équipe représente $28,2 \%$ des répondants en premier degré contre $50,2 \%$ en second degré $(p<0,01)$, et les outils de communication privilégiés sont le mél. $(84,1 \%)$ et le texto $(49,4 \%)$. L'usage d'applications de travail collaboratif $(9,4 \%)$ ou d'un environnement numérique de travail (ENT) reste faible, et ce, même dans le second degré, où seuls $58,5 \%$ des répondants l'utilisent pour travailler entre collègues malgré la présence d'un ENT dans toute l'académie.

\section{LE TRAVAIL COLLECTIF CENTRÉ SUR L'ÉLÈVE : UNE TYPOLOGIE D'ACTIVITÉS VARIABLE SELON LES DEGRÉS}

Les enseignants de l'échantillon déclarent, pour $55 \%$ d'entre eux, que le travail en groupe, lorsqu'il est mis en place, dure généralement le temps d'une séance entière (30 à 60 minutes). Si pour $73,7 \%$ des répondants la taille idéale d'un groupe est de 3 à 4 élèves, il est à noter que pour $13,7 \%$ des enseignants du collège (contre 2,9\% seulement dans le premier degré), la taille d'un groupe idéal correspond à un grand groupe, voire une demi-classe (8 à 15 élèves). La notion de travail collectif prend donc des représentations variables selon les personnes et les niveaux. Le travail collectif extrascolaire des élèves n'est pas marginal, puisque 16,7 à $25 \%$ des enseignants le demandent respectivement pour l'école et le collège. Au cours du travail collectif, les tâches au sein du groupe sont réparties dans $53,7 \%$ des cas par les élèves et demeurent identiques pour tous dans $92,3 \%$ des cas. II s'agit d'un temps au cours duquel les enseignants évaluent surtout les élèves de manière formative et aussi collective pour respectivement $50,4 \%$ et $58,8 \%$ des répondants. II faut noter que, si les enseignants du collège évaluent également les apprentissages de manière sommative pour $39,7 \%$ d'entre eux, ils ne sont que $14,7 \%$ à le faire à l'école $(p<0,01)$. De la même façon, les enseignants du premier degré ne sont que $18,3 \%$ à évaluer les élèves individuellement au cours des travaux de groupe, contre $45,3 \%$ des enseignants de collège $(p<0,01)$.

Les proportions de typologies d'activités conduites en groupe et les objectifs du socle poursuivis dans ce cadre diffèrent également entre les deux degrés, et ce, souvent de façon très significative (voir figures 2 et 3). En effet, les activités de groupe poursuivies en premier degré sont plus diversifiées qu'en second degré : quatre activités plébiscitent en particulier l'entraide, la résolution de problèmes, les activités d'exploration et d'expérimentation, et dépassent plus de $65 \%$ des déclarations pour ce public des écoles. 
Figure 2

Répartition des types d'activités de groupe en classe chez les enseignants des $1^{\text {er }}$ et $2^{\mathrm{e}}$ degrés

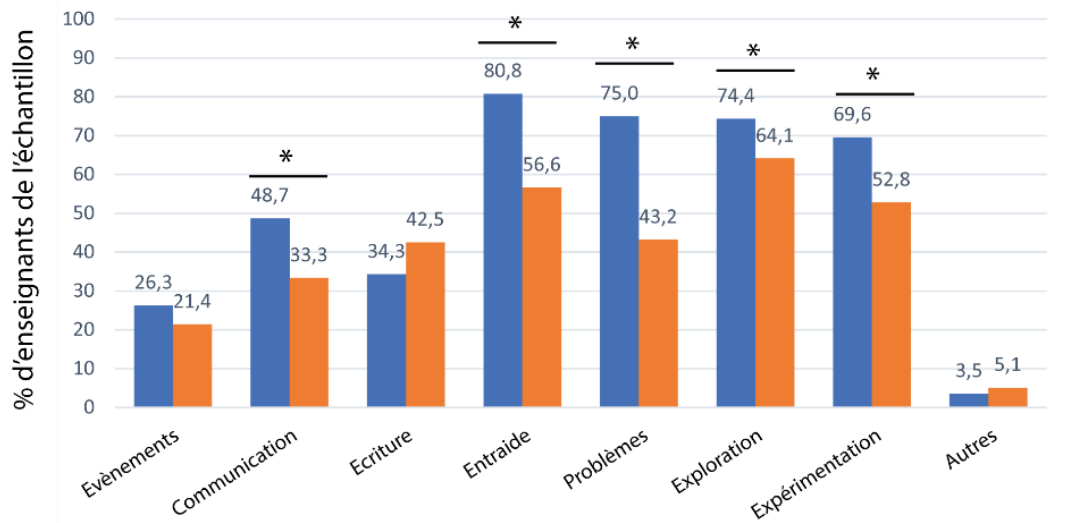

Enseignants

du 1er degré

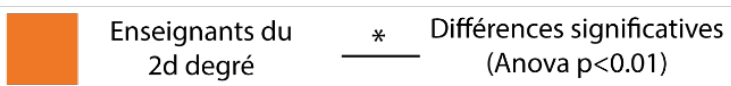

Figure 3

Répartition des objectifs visés par les activités en groupe dans le socle commun de compétences chez les enseignants des $1^{\text {er }}$ et $2^{e}$ degrés

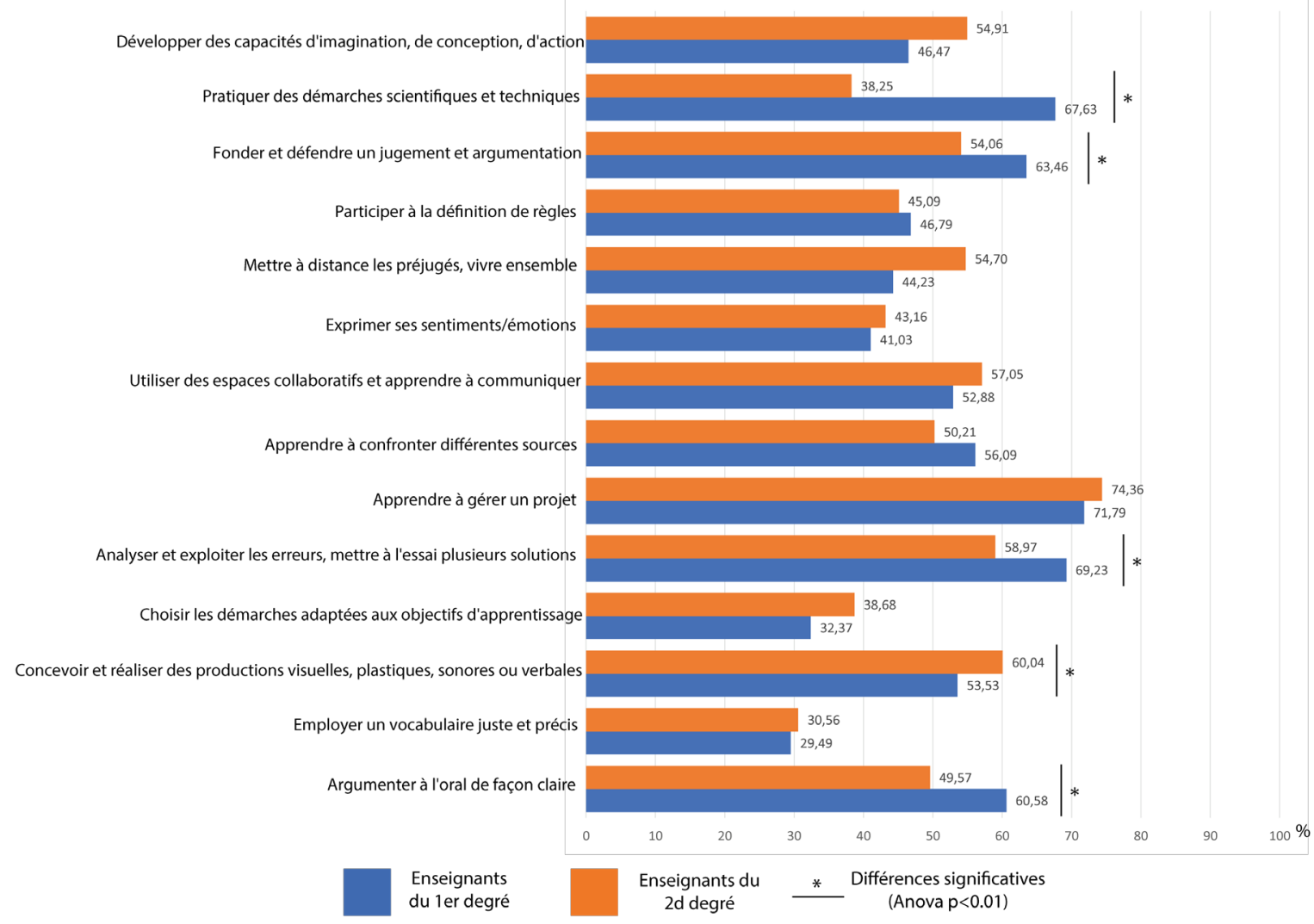


Les ressources au format papier restent le type de support privilégié par $87,5 \%$ des répondants de l'échantillon cible, essentiellement via des documents (84\%), des livres et manuels $(76 \%)$ et le dictionnaire (60 \%), mais aussi des jeux et du matériel pédagogique (27,6\%). 59,2 \% proposent également des supports numériques, mais ce sont essentiellement des documents au format numérique $(51,4 \%)$ ou l'accès au Web.

Enfin, à la question sur la fréquence du travail en groupe, la figure 4 montre que les travaux collectifs entre élèves sont significativement plus fréquents dans le primaire $(p<0,01)$, avec $75 \%$ des professeurs des écoles déclarant faire travailler leurs élèves en groupe au moins une fois par semaine, contre $53,4 \%$ au collège.

\section{Figure 4}

Fréquence déclarée du travail en groupe chez les enseignants des $1^{\text {er }}$ et $2^{e}$ degrés

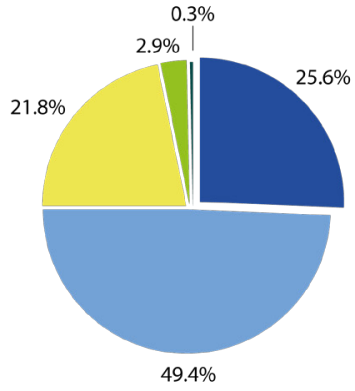

Enseignants du 1er degré

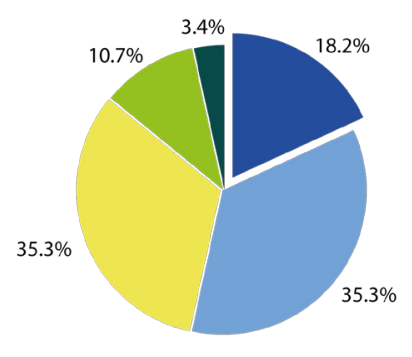

Enseignants du 2 d degré

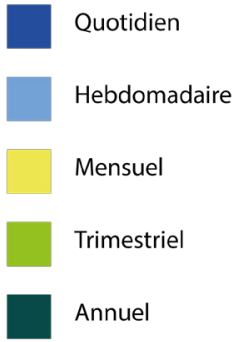

Annuel

\section{Une catégorisation des pratiques en deux grands ensembles de variables}

Dans un premier temps, la CAH en composantes multiples permet de distinguer deux grands ensembles de variables, appelés « clusters $A$ et $B$ », regroupant respectivement 65 et 55 variables représentées dans les figures 5 et 6 . Chaque famille de variables y a été marquée à partir d'un code couleur arbitraire associé à huit grandes catégories de questions, pour en faciliter la lecture et l'interprétation. $\mathrm{La} C A H$, en définissant un niveau de coupe à 2,0, permet une partition des variables en quatre classes nommées "clusters A1, A2, B1 et B2 » incluses deux à deux dans les ensembles précédents. 


\section{Figure 5}

Aperçu général des clusters $A$ issus de la $\mathrm{CAH}$, avec indication (en rouge) du pourcentage de répondants (issu des tris à plat) pour chaque variable classée

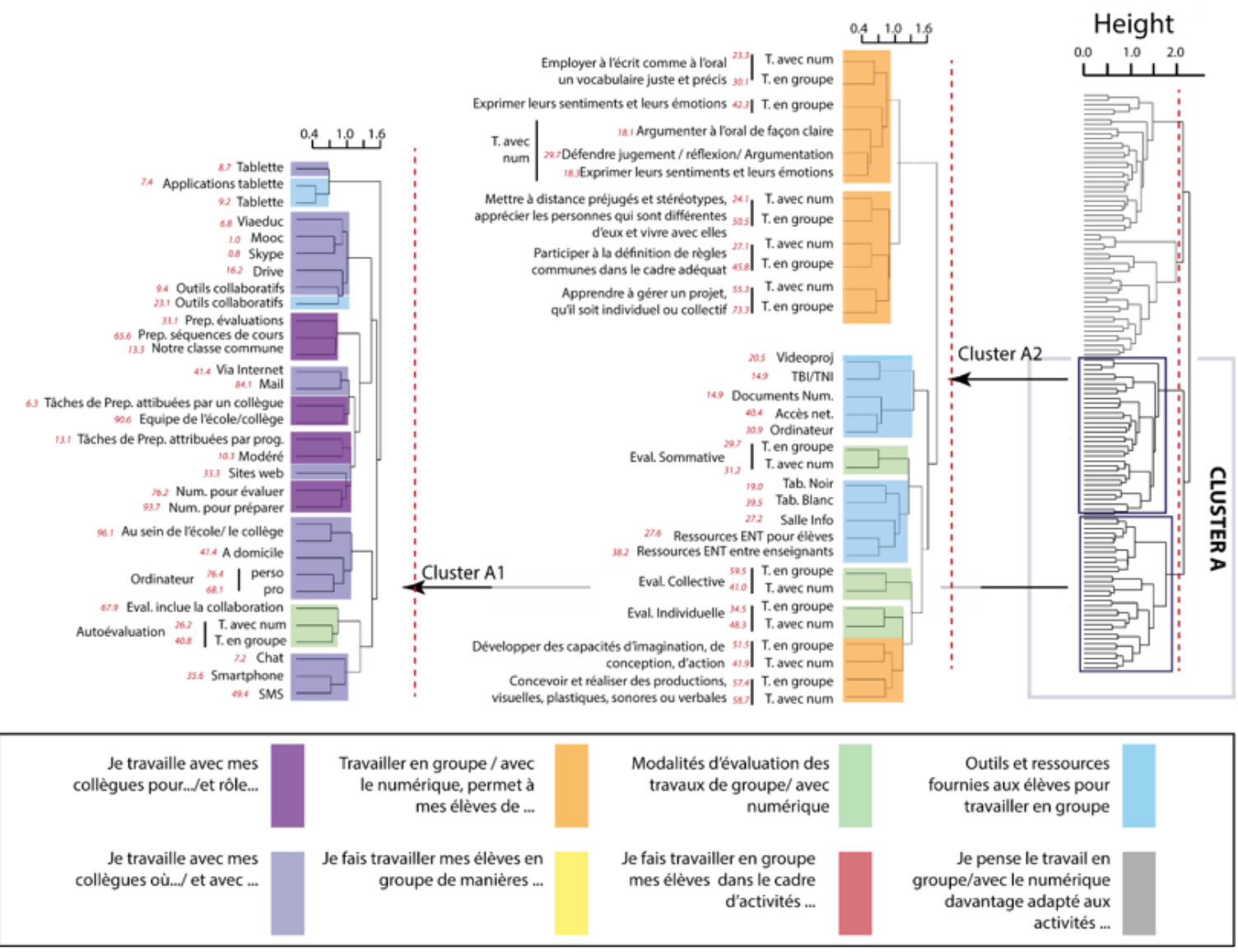

Note. Abréviations et sigles utilisés : T (travail), Num (numérique), Prep (préparations), Eval (évaluations), net (Internet), Tab (tableau), videoproj (vidéoprojecteur), Dico (dictionnaire), Res (résolution), ENT (environnement numérique de travail), TBI (tableau interactif), TNI (tableau numérique interactif).

Le cluster A1 agrège des variables associées essentiellement aux activités professionnelles pour préparer sa classe avec différents outils, ressources, contenus et supports numériques, personnels ou professionnels, et avec différents lieux où s'effectue ce travail entre pairs (domicile, établissement). Ce cluster explique $25,5 \%$ de la variance totale des réponses.

Le cluster A2 regroupe des variables d'objectifs d'apprentissage du socle commun"1 de compétences, de connaissances et de culture (cycle 3) essentiellement associables aux domaines 1 (les langages pour penser et communiquer) et 2 (les méthodes et outils pour apprendre) ainsi qu'aux dimensions du vivre ensemble et de la construction du statut d'élève du domaine 3 (la formation de la personne et du citoyen). Ils sont associés statistiquement avec des modalités d'évaluations sommatives de bilan d'acquis

\footnotetext{
${ }^{11} \mathrm{Cf}$ : socles communs de connaissances, compétences et culture en fin de cycle 3 (https://cache.media.eduscol.education.fr/file/College 2016/74/4/RAE Evaluation socle cycle 3 643744.pdf, consulté le 10/06/2020) et de cycle 4 (https://cache.media.eduscol.education.fr/file/College 2016/74/6/RAE Evaluation socle cycle 4 643746.pdf, consulté le 10/06/2020).
} 
individuels ou collectifs, ainsi qu'avec différents outils, ressources et espaces disponibles dans la classe ou à l'école (qu'ils soient numériques ou non). Ce cluster explique $29,6 \%$ de la variance totale.

\section{Figure 6}

Aperçu général des clusters $B$ issus de la $\mathrm{CAH}$ avec indication (en rouge) du pourcentage de répondants (issu des tris à plat) pour chaque variable classée

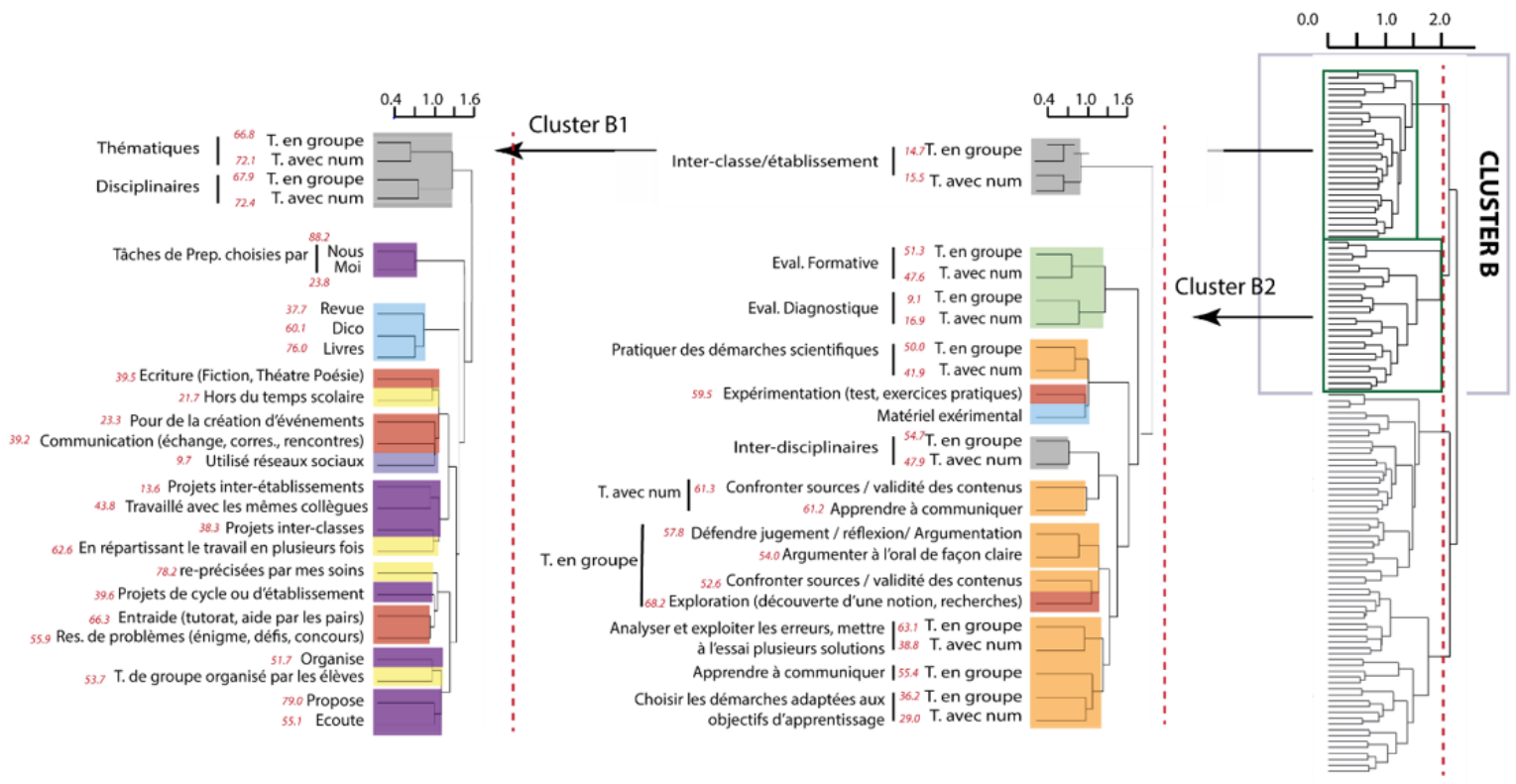

Le cluster B1 semble le plus hétérogène. II regroupe des variables liées aux activités de conception entre collègues sur des projets communs avec des activités thématiques ou disciplinaires en lien avec la culture, la communication, les échanges, l'entraide et la résolution de problèmes, et avec des modalités de travail collectif au sein des groupes d'élèves ou d'enseignants. Ce cluster explique $24,5 \%$ de la variance totale.

Le cluster B2 associe enfin des variables d'objectifs d'apprentissage visant essentiellement à donner les fondements de la résolution de problèmes, de la construction de l'esprit critique et de la vie en société. Ces objectifs sont associables aux domaines 3, 4 (les systèmes naturels et les systèmes techniques) et 5 (les représentations du monde et de l'activité humaine) du socle commun avec des activités interdisciplinaires associées à l'expérimentation et à l'exploration. Ils reposent sur des modalités d'évaluation diagnostique et formative visant à (faire) comprendre et analyser les besoins des élèves. $\mathrm{Ce}$ cluster explique $20,5 \%$ de la variance totale.

\section{Deux catégories principales de variables prédictives du travail collectif en classe}

Dans un second temps, la régression PLS permet d'identifier les catégories de variables contributives à l'explication des différences de fréquence de travail en groupe en classe, pour laquelle des différences ont déjà été repérées lors des tris à plat (voir figure 4). Ce nouveau traitement statistique des données permet un éclairage comparatif selon les deux niveaux d'enseignement, que nous détaillons ci-dessous. Dans les deux cas, deux catégories de variables sur six apparaissent comme les plus contributives (avec des indices de contribution relative de 47,1 et 42,8 pour l'école, et de 42,7 et 27,3 pour le collège) : 
- catégorie C5: instrumentalité perçue du travail en groupe pour favoriser l'émergence de compétences du socle commun;

- catégorie C4 : types d'activités de travail en groupe réalisées.

\section{À L’ÉCOLE}

Sur 66 variables mises dans l'analyse, 27 variables ont un lien significatif $(p<0,05)$ avec la fréquence déclarée du travail en groupe et expliquent $16,5 \%\left(R^{2}\right)$ de la variance de celle-ci. Pour chacune des variables et des catégories de variable, nous avons établi, à partir des corrélations et des bêtas de régression, un indice de contribution relative (ICR) au $\mathrm{R}^{2}$. Comme la somme des ICR est égale à $100 \%$ du $R^{2}$, il est alors possible de hiérarchiser les variables et leurs catégories en fonction de la taille des liens avec le critère. Le tableau 2 montre les catégories et leurs variables hiérarchisées selon I'ICR. Deux catégories de variables sur six sont les plus contributives avec un ICR de 47,1 et 42,8 respectivement : ce sont les catégories C5 (relatives au socle de compétences) et C4 (types d'activités de travail en groupe).

Ainsi, plus les professeurs des écoles sont persuadés que le socle de compétences peut être amélioré par le travail en groupe, voire l'usage du numérique, plus ils déclarent une fréquence élevée de celui-ci. Parmi les 14 compétences qui semblent les plus promouvables par le travail en groupe, 12 ressortent comme significativement liées. Mais trois se distinguent particulièrement en termes d'ICR : V3.a, V3.b et V3.c avec un ICR supérieur à 4,5 qui expriment cette vision que le travail en groupe permet d'apprendre à s'organiser avec les autres, à atteindre un objectif assigné et à respecter les uns et les autres dans le groupe. Quant à elles, les variables de la catégorie C4 traduisent qu'une expérience soutenue dans une diversité d'activités avec des groupes et un jugement positif de l'enseignant sur la réussite probable de divers décloisonnements facilitent la répétition au cours du temps du choix d'un travail en groupe d'élèves (V4 et V5, ICR global de 20,4). Trois activités sont par ailleurs plus favorables à l'augmentation de cette fréquence : elles renvoient à des activités d'appropriation de nouveaux savoirs, de mises en situation, à la résolution de problèmes ou à des activités de découverte (V6.a/b/c avec un ICR global de 13,7).

Les autres catégories de variables ont une influence largement plus faible (ICR global à 10,1). On peut noter que la fréquence de collaboration avec les collègues et le partage de supports numériques entre eux ont probablement un rôle facilitateur sur le travail en groupe d'élèves (V7 et V8, ICR global de 7,5).

\section{Tableau 2}

Effet des variables [ICR] selon leur catégorie d'appartenance sur la fréquence de travail en groupe à l'école

Catégories de variable

\begin{tabular}{l}
\hline \\
C5 : Instrumentalité \\
perçue du travail en \\
groupe pour \\
favoriser \\
l'émergence de \\
compétences \\
[ICR total $=47,1]$
\end{tabular}

Variables (V) contributives à l'explication des différences de fréquence de travail en groupe [ICR]

V1: Être persuadé que plusieurs compétences sont améliorables par le travail en groupe $[10,3]$

V2 : $\quad$ Être persuadé que de nombreuses compétences sont améliorables par le travail en groupe ET par l'usage du numérique [5,2]

Avoir coché une ou des compétences ci-dessous comme étant favorisées par le travail en groupe :

V3.a : Choisir les démarches adaptées aux objectifs d'apprentissage préalablement explicités $[5,5]$

V3.b : Mettre à distance préjugés et stéréotypes, apprécier les personnes qui sont différentes d'eux et vivre avec elles [5,2] 


\begin{tabular}{|c|c|}
\hline $\begin{array}{l}\text { Catégories de } \\
\text { variable }\end{array}$ & $\begin{array}{l}\text { Variables }(\mathrm{V}) \text { contributives à l'explication } \\
\text { des différences de fréquence de travail en groupe [ICR] }\end{array}$ \\
\hline & 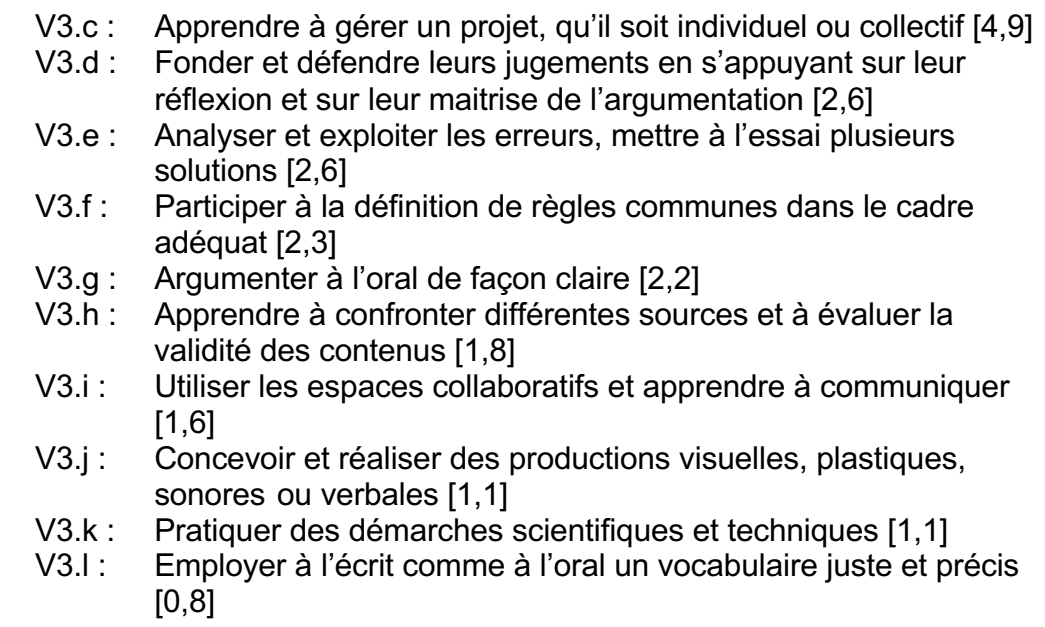 \\
\hline $\begin{array}{l}\text { C4: Types } \\
\text { d'activités de travail } \\
\text { en groupe réalisées } \\
{[\text { ICR total }=42,8]}\end{array}$ & 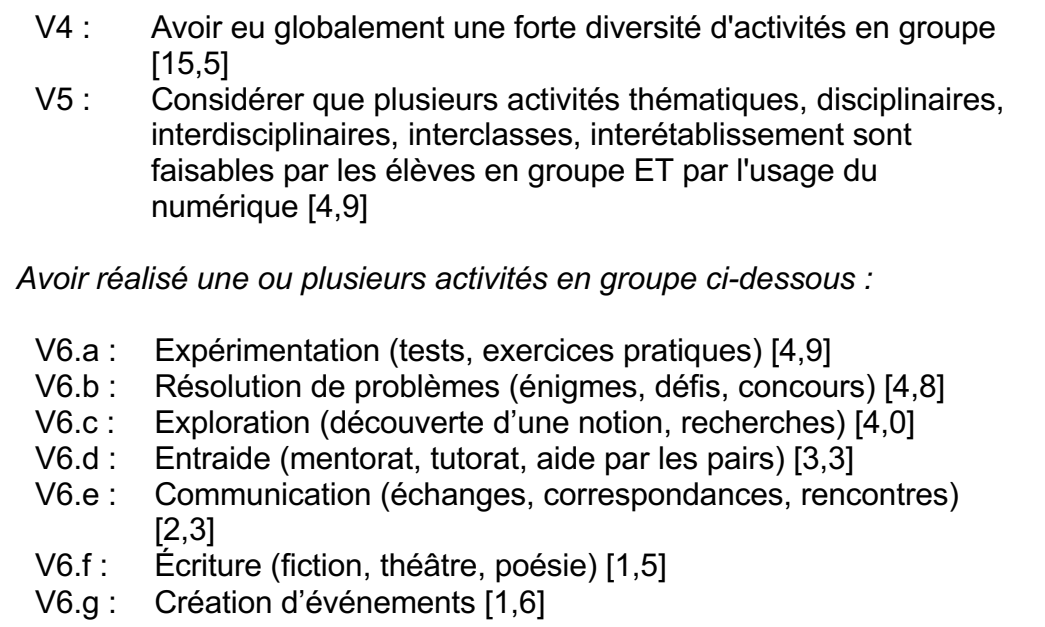 \\
\hline $\begin{array}{l}\text { Autres catégories } \\
\text { C1, C2 et C3 } \\
{[\text { ICR total }=10,1]}\end{array}$ & 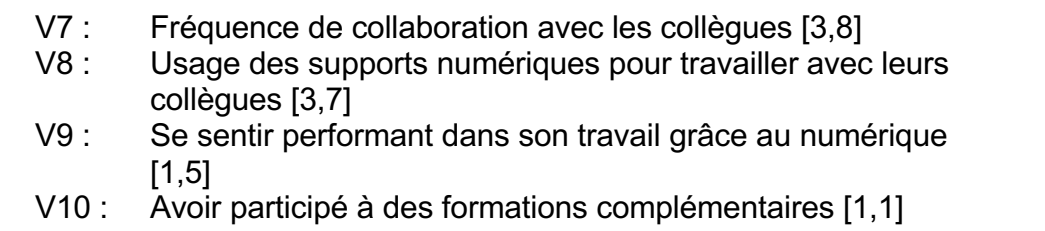 \\
\hline
\end{tabular}

\section{AU COLLÈGE}

Trente-deux variables significatives ont été retenues, prenant en compte $19,5 \%$ de la variance. II y a donc un meilleur ajustement de la fréquence du travail en groupe pour les enseignants en collège que pour ceux des écoles. Cela est d'ailleurs confirmé par les calculs de $\mathrm{R}^{2}$ ajustés en fonction des effectifs des groupes et du nombre de prédicteurs : le $\mathrm{R}^{2}$ ajusté est de $10,4 \%$ pour les enseignants du primaire contre un $\mathrm{R}^{2}$ ajusté de $14,4 \%$ pour les enseignants du collège. La diversité des catégories de variables ayant une influence est aussi plus étendue dans le tableau 3 des résultats. 
En effet, si les catégories $\mathrm{C} 4$ et $\mathrm{C} 5$ ont toujours une influence prépondérante, les catégories relatives au type de collaboration avec les collègues (C3) et l'attitude sur les technologies du numérique (C2) sont ici mieux représentées que pour les enseignants du premier degré (ICR global des deux dernières catégories égales à 20,0 contre 9,0 dans les écoles). La catégorie C4 est maintenant première par la taille de son influence $(I C R=42,7)$. On retrouve l'importance d'avoir eu une diversité d'activités en groupe et la confiance dans des décloisonnements favorables au travail collectif des élèves au côté des moyens numériques (ICR de 16,0 et 5,2 respectivement). L'ordre des activités n'est pas identique à celui des écoles : les exercices pratiques sont toujours en premier (V6.a, ICR $=9,1)$, mais ils sont suivis par des aspects relatifs à la constitution de réseaux sociaux de classes (V6.e, ICR $=4,3$ ) ou de supports sociaux d'entraide par les pairs (V6.d, ICR $=3,0)$.

La catégorie C5 est présente en deuxième position (ICR $=27,3)$ : c'est parce que les enseignants de collège sont persuadés du lien entre développement des compétences et travail en groupe que l'on peut observer de manière concomitante une fréquence de ce travail plus élevée dans leurs classes (V1 et V2 : ICR de 5,9 et 3,1 respectivement). Par contre, l'ordre des compétences du socle commun n'est pas le même que dans le premier degré et de nouvelles compétences apparaissent ici comme les plus en lien avec cette fréquence du travail en groupe :

- $\quad$ apprendre à confronter des sources $(V 3 . h, I C R=3,4)$;

- $\quad$ exprimer ses émotions (V3.m, non présente pour les écoles, ICR = 2,6);

- choisir des façons de faire adaptées (V3.a, ayant moins d'importance pour les écoles, ICR = 2,1);

- développer des capacités d'imagination (V3.n, non présente pour les écoles, ICR = 2).

La fréquence et la diversité des collaborations avec les collègues ainsi qu'une certaine aisance dans l'usage des technologies du numérique sont des vecteurs favorisant quelque peu le travail en groupe (C3, $C 2$; ICR = 20). Enfin, des éléments du contexte professionnel révèlent de possibles différences entre les enseignants du secondaire, toutes choses égales par ailleurs : les enseignants en technologie auraient tendance à déclarer un travail collaboratif des élèves plus fréquent $(I C R=5,2)$, ce qui est en accord avec les attentes des programmes scolaires dans cette discipline marquée par le travail en groupe projet, alors que les enseignants en mathématiques tendent à légèrement moins le pratiquer que dans les autres matières (ICR $=2,1$ : effet négatif). De manière très anecdotique, la catégorie C6 (ressources matérielles et numériques) est représentée uniquement et faiblement par une seule variable V14 (avoir du matériel expérimental), très modeste marqueur du travail en groupe seulement au collège (ICR $=1,5)$.

\section{Tableau 3}

Effet des variables [ICR] selon leur catégorie d'appartenance sur les différences de fréquence de travail en groupe au collège

\begin{tabular}{|c|c|}
\hline Catégories de variable & $\begin{array}{l}\text { Variables }(\mathrm{V}) \text { contributives à l'explication } \\
\text { des différences de fréquence de travail en groupe [ICR] }\end{array}$ \\
\hline $\begin{array}{l}\text { C4 : Types d'activités de } \\
\text { travail en groupe réalisées } \\
{[\text { ICR total }=42,7]}\end{array}$ & $\begin{array}{cl}\text { V4: } & \begin{array}{l}\text { Avoir eu globalement une forte diversité d'activités en groupe } \\
{[16,0]}\end{array} \\
\text { V5: } & \begin{array}{l}\text { Considérer que plusieurs activités thématiques, disciplinaires, } \\
\text { interdisciplinaires, interclasses, interétablissement sont } \\
\text { faisables par les élèves en groupe ET par l'usage du } \\
\text { numérique }[5,2]\end{array} \\
\text { Avoir réalisé une ou plusieurs activités en groupe ci-dessous : } \\
\text { V6.a : Expérimentation (tests, exercices pratiques) }[9,1]\end{array}$ \\
\hline
\end{tabular}




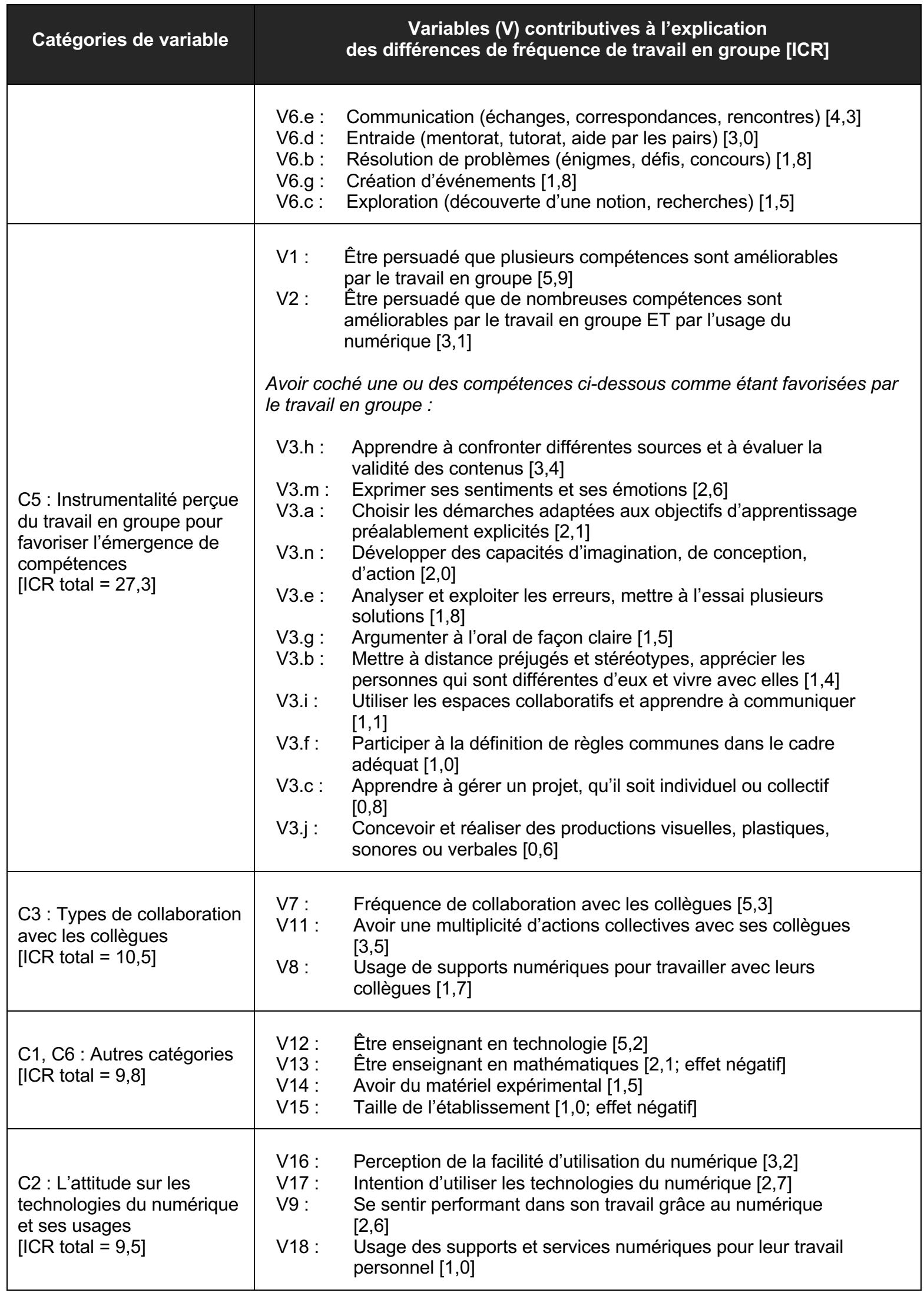




\section{Discussion}

Avant toute chose, on note ici que la très grande majorité des enseignants interrogés déclare une pratique collective effective et régulière, à la fois entre pairs et au sein de leurs classes, marquant ainsi une évolution avec les travaux de Dupriez datant de 2010 et qui parlait de récit mythique et de distance entre l'injonction au travail en équipe et les pratiques effectives à l'école en France. Notre enquête permet donc de franchir un palier en offrant une description fine des pratiques quotidiennes déclarées par les enseignants en cycles 3 et 4 , et en identifiant une nouvelle catégorisation de ces pratiques.

\section{Une catégorisation des pratiques selon plusieurs axes paradigmatiques saillants}

La figure 7 présente les mêmes résultats issus de la CAH (voir figures 5 et 6 ), mais sous forme de diagrammes d'Euler (avec vue de dessus), et en conservant les mêmes codes couleurs pour identifier les huit catégories de questions posées durant le questionnaire. Pour cette analyse davantage interprétative, nous y représentons les quatre clusters A1, A2, B1 et B2 en quatre pôles positionnés sur les deux axes paradigmatiques suivants (voir figure 7):

- $\quad$ axe paradigmatique horizontal : cluster centré sur l'enseignant vs cluster centré sur l'apprenant;

- $\quad$ axe paradigmatique vertical : cluster autocentré (sur la classe) vs cluster hétérocentré (sur les autres et l'extérieur). 
Figure 7

Catégorisation des pratiques de travail collectif des enseignants interrogés par axes paradigmatiques

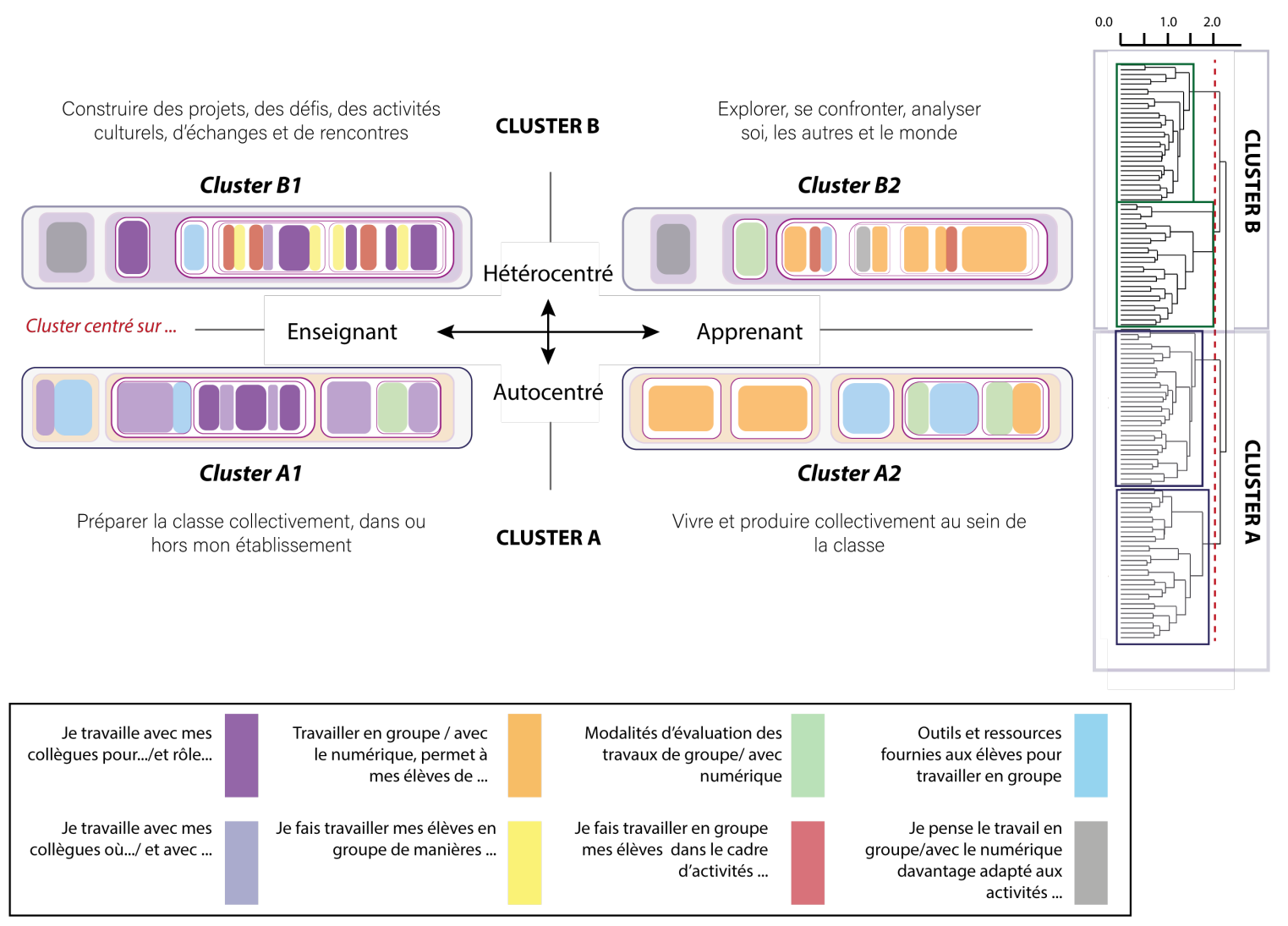

Avant d'entrer ci-après dans l'interprétation des deux axes paradigmatiques ainsi identifiés, il est intéressant de constater que la participation des quatre clusters à la variance totale apparaît relativement homogène avec des pourcentages allant de 20,5 à $29,6 \%$ selon les cas. Il est cependant important de mentionner ici la portée de ces regroupements de variables dans les réponses du questionnaire : ils permettent de catégoriser des formes de pratiques enseignantes de travail collectif entre pairs et en classe, mais ne sont pas exclusifs les uns des autres. Concrètement, cela signifie qu'un même répondant peut tout à fait appartenir à plusieurs clusters et à des degrés divers.

\section{AXE ENSEIGNANT VS APPRENANT}

Nous restons prudents sur le biais possible de la structure du questionnaire sur l'émergence d'un tel axe, étant donné sa répartition en deux sous-ensembles de questions: l'un sur le travail collectif entre enseignants et son contexte professionnel (volets « $a$ » et « $b$ »), l'autre sur le travail collectif des élèves et les outils de la classe (volets « $d$ » et « $e$ »). Car une telle répartition peut mathématiquement peser sur la proportion des variables regroupées par la $\mathrm{CAH}$ et appartenant à chacun de ces sous-ensembles, même si leur regroupement final demeure significatif en soi. En effet, ce premier axe horizontal rejoint la distinction faite par Cristol (2017) entre deux formes de sociabilités d'apprentissage : le point de vue de l'apprenant (ou " sociodidaxie ») ou celui du tiers (" pairagogie »). La première correspond à de nouvelles formes de sociabilités autodidactiques par lesquelles l'apprentissage est co-construit avec les autres pour un enjeu commun, la seconde se traduit par un savoir-faire éducatif appliqué à l'apprentissage par les pairs qui se déploie par la mise en commun, le partage, le collectif, la coopération, le collaboratif, voire la coopétition. 
Ici, les clusters A1 et B1 regroupent majoritairement des variables décrivant les modalités du travail entre pairs : " Je travaille avec mes collègues pour.../et rôle... ", " Je travaille avec mes collègues où.../et avec... ». Le cluster A1 illustre une communauté enseignante orientée sur la préparation et la conception de séquences de cours (65,6\% des répondants), majoritairement réalisées en équipe $(90,6 \%)$ et au sein des établissements $(96,1 \%)$. Le cluster B1 met davantage en exergue la nature et les modalités de la collaboration : projets thématiques ou disciplinaires (66,8 et $67,9 \%$ des répondants), répartition des tâches dans le travail collectif (proposer, écouter, organiser), entraide par les pairs $(66,3 \%)$. On peut ici les mettre en miroir avec deux des six manières de nommer le travail entre enseignants que nous avions citées chez Vangrieken et al. (2015): le cluster A1 représente les teacher teams (appartenance à une équipe pédagogique) tandis que le cluster B1 se rapporte davantage aux communities of practice (incluant entraide mutuelle, collaboration et collégialité au sein d'un groupe professionnel). Comme le soulignent Spillane et al. (2016), ces formes du travail collectif se positionnent d'abord en amont sur le contenu d'enseignement et peuvent potentiellement toucher différentes dimensions :

- la progression des séances pédagogiques, leur planification, le rythme d'enseignement;

- le curriculum et les partenariats intercycles (préparation des leçons, échange sur le contenu, montage d'un projet);

- l'expérimentation de nouveaux outils, de nouvelles activités (dialogues ciblés entre élèves) ou le développement de nouveaux matériels didactiques;

- la mise en place d'un nouveau programme;

- des discussions sur l'éventail des stratégies d'enseignement et d'apprentissage visant à répondre aux besoins particuliers des élèves;

- l'élaboration d'évaluations conjointes.

Quant aux clusters A2 et B2, ils regroupent des variables axées sur les objectifs d'apprentissage et les modalités du travail collectif entre élèves : «Travailler en groupe/avec le numérique permet à mes élèves de... ", " Modalités d'évaluation des travaux de groupe/avec numérique », "Outils et ressources fournies aux élèves pour travailler en groupe ». Chacun comprend une sélection de compétences issues du socle commun et considérées par les répondants comme des objectifs d'apprentissage du travail collectif (nous ne mentionnons ci-dessous que celles dont le pourcentage de répondants est > à 50 et listées par ordre d'importance) :

- cluster A2 : apprendre à gérer un projet, concevoir et réaliser des productions, développer des capacités d'imagination, de conception et d'action, mettre à distance préjugés et stéréotypes, apprécier les personnes qui sont différentes d'eux et vivre avec elles;

- cluster B2 : analyser et exploiter les erreurs, mettre à l'essai plusieurs solutions, défendre un jugement, une réflexion, une argumentation, apprendre à communiquer, argumenter à l'oral de façon claire, confronter les sources et la validité des contenus, pratiquer des démarches scientifiques.

Ces deux clusters A2 et B2 se réfèrent davantage à des conceptions différentes de la notion de collaboration, comme les distingue Baudrit (2007), même s'il les rattache pour sa part à des zones géographiques et culturelles différentes (Europe et Amérique du Nord) :

- une collaboration contradictoire (que nous observons dans le cluster B2) : orientation européenne, où l'accent est mis sur le processus cognitif. Ce n'est pas tant le résultat final qui importe que le raisonnement, le cheminement des élèves. L'autonomie des sujets est primordiale. Le groupe est autonome et se construit « chemin faisant »;

- une collaboration constructive (qui correspond davantage au cluster A2) : orientation nordaméricaine, où la résolution de la tâche est essentielle. Le groupe est préalablement organisé par l'enseignant ou le formateur, chacun ayant un rôle défini à remplir. 
Ces deux clusters orientés sur l'apprenant soulignent l'importance du socle commun de compétences, de connaissance et de culture comme document de référence par rapport auquel les répondants justifient leurs choix pédagogiques. Nous pouvons parler ici d'une culture professionnelle et organisationnelle partagée (Maubant, 2014) qui, à travers la prise en compte de la communauté apprenante, illustre également les conceptions et valeurs professionnelles de la communauté enseignante. Sur ce dernier point, il est intéressant de noter que ces conceptions des pratiques éducatives peuvent différer sans forcément s'opposer, étant donné qu'un même enseignant peut tout à fait appartenir à plusieurs clusters parmi ceux que nous avons identifiés ici. À la différence de Dupriez (2010), qui évoquait des dichotomies entre mission d'instruction et mission d'éducation, ou entre pratiques pédagogiques divergentes (comme dans le cluster B2) ou convergentes (comme dans le cluster A2), cette diversité des formes d'actions collectives n'est donc pas forcément discriminante et peut se révéler parfois complémentaire.

\section{AXE AUTOCENTRÉ VS HÉTÉROCENTRÉ}

Ce deuxième axe vertical nous permet de différencier deux autres formes de catégorisation des pratiques du travail collectif, dont l'effet, cette fois-ci, ne peut pas être attribué à la structure du questionnaire. Les clusters A sont orientés sur le travail collectif autocentré, où il s'agit de coopérer et de produire au sein de la classe, et les clusters B sur le travail collectif hétérocentré, où l'objectif d'apprentissage est de collaborer, de s'ouvrir, de se confronter aux autres et au monde extérieur à la classe. Pour faire à nouveau le lien avec les dichotomies évoquées précédemment chez Dupriez (2010), on retrouve ici sa distinction entre apprentissages décontextualisés (clusters A centrés sur la classe) ou contextualisés (clusters B tournés vers l'extérieur), où les seconds valorisent davantage les approches interdisciplinaires et la capacité à agir dans un environnement réel.

Dans les clusters A1 et A2, nous rejoignons également l'orientation de la collaboration dite constructive telle que la définit Baudrit (2017), où la préparation des séquences et les objectifs d'apprentissage sont orientés sur les résultats à produire et s'appuie sur des compétences de gestion de projets et de conception/production, et des modalités d'évaluation majoritairement collectives (59,5\% des répondants). Dans les clusters B1 et B2, c'est davantage l'orientation de la collaboration dite contradictoire qui prime, où le processus cognitif (analyser, confronter, argumenter, défendre un jugement) et la réflexivité des élèves (avec évaluation formative pour $51,3 \%$ des répondants) dominent. Les compétences visées s'y inscrivent davantage dans le vivre ensemble tel qu'on les retrouve dans le domaine 3 du socle commun ( "La formation de la personne et du citoyen »).

Cet axe illustre finalement les trois perspectives de l'apprentissage collaboratif et leurs questions associées telles que les identifie Orellana (2005):

- perspectives technico-pédagogiques pour les groupes : quel moyen adopter pour réussir?

- perspectives sociales et communautaires pour l'école : comment créer un esprit communautaire améliorant le vivre ensemble?

- perspectives philosophico-éducatives pour la société où la communauté d'apprentissage serait liée à la qualité d'être et à la réalisation de l'être humain comme être pensant et réflexif, visant la construction de relations authentiques avec les autres.

\section{Le poids relatif des épistémologies personnelles}

Concernant les résultats obtenus par la régression PLS, nous pouvons remarquer l'importance des croyances chez les enseignants dans l'une des deux principales catégories de variables (dont l'ICR est parmi les plus élevés, que ce soit pour l'école -47,1 - ou pour le collège - 27,3-) : l'instrumentalité perçue du travail en groupe pour favoriser l'émergence de compétences. En effet, cette catégorie est constituée 
de variables contributives fondées sur des perceptions chez les répondants : « être persuadé que plusieurs compétences sont améliorables par le travail en groupe " et "être persuadé que de nombreuses compétences sont améliorables par le travail en groupe et par l'usage du numérique ». Elle découle des questions de l'enquête en ligne formulées comme suit : " Je pense que travailler en groupe permet à mes élèves de... " (liste de compétences du socle à cocher) et " Je pense que travailler avec le numérique permet à mes élèves de... » (même liste).

La contribution importante de ces variables aux différences de fréquence du travail en groupe en classe permet de souligner l'impact des épistémologies personnelles des enseignants comme système de croyances construites par eux et qui orientent leurs actions. Selon Poteaux (2017, p. 26-27), ces croyances ou représentations, ou autres termes répertoriés tels que conception, savoir enseignant ou épistémologie personnelle, désignent la représentation qu'une personne se fait de la réalité construite par l'expérience et qui possède assez de validité, de vérité, de crédibilité pour guider la pensée ou le comportement. Selon l'auteure, les croyances sont d'abord personnelles, mais peuvent être partagées au sein d'un groupe social ou professionnel. Elles s'installent et résistent tant qu'elles ne sont pas déstabilisées par des contreargumentations rigoureuses et justifiées. Elles portent essentiellement sur les apprenants, l'enseignement, l'apprentissage, la façon d'être efficace, les contenus disciplinaires, la relation aux collègues et à l'institution, et se traduisent dans le discours par des énoncés commençant par " je crois que ", " je pense » ou par des métaphores. Selon Abric (2003, cité par Poteaux, 2017), les croyances peuvent remplir plusieurs fonctions :

- épistémiques : donner un sens aux expériences vécues, faire état de ses certitudes, comprendre le sens des innovations, réajuster ses croyances le cas échéant;

- identitaires : se situer dans le champ social et professionnel, affirmer une appartenance à un groupe, à un métier, à une institution;

- normatives : orienter les comportements, les actions et les pratiques;

- justificatrices ou autodéfensives.

Ce résultat est important pour favoriser la dissémination potentielle des usages pédagogiques des interfaces de nouvelle génération conçues dans notre projet e-TAC au-delà des partenaires actuels, car il souligne qu'il faudra travailler au préalable sur les croyances actuelles des enseignants vis-à-vis des apports du numérique et du travail en groupe pour améliorer les apprentissages de leurs élèves. En effet, les catégories de variables contributives à l'explication des différences de fréquence de travail en groupe en classe montrent que plus les enseignants pensent que le travail collectif permet la diversification de leurs activités pédagogiques et permet l'apprentissage des compétences du socle, plus ils mobilisent le travail collectif.

\section{Une place du numérique à relativiser fortement}

Pour finir, nous relevons un autre résultat significatif de notre enquête et qui concerne la place du numérique dans les pratiques de travail collectif entre pairs et avec les élèves. Malgré la présence de nombreuses questions dans l'enquête sur le type d'outils et de ressources numériques utilisés pour ce travail collectif, leurs modalités d'usage et leur rôle dans l'acquisition et l'évaluation de compétences du socle commun, force est de constater que leur poids statistique demeure très limité, que ce soit dans les résultats de la CAH ou dans ceux de la régression PLS. 
Dans les quatre clusters de la $\mathrm{CAH}$, les outils et ressources numériques apparaissent ainsi de trois façons :

- en négatif : c'est leur très faible taux de présence dans les réponses qui explique le regroupement des variables primaires, et non l'inverse. C'est particulièrement le cas dans le cluster B1, où il n'apparaît qu'une seule fois pour l'usage des réseaux sociaux ( $9,7 \%$ des répondants);

- en association avec la modalité du travail en groupe: la majorité des compétences du socle contribuant à la formation des clusters sont associées à la fois au travail en groupe et au travail avec le numérique, même si l'on peut constater que les pourcentages de réponses sont largement supérieurs pour la première modalité;

- les seules exceptions à cette règle d'association concernent les trois compétences du socle suivantes, où le travail avec le numérique présente des pourcentages plus élevés que le travail en groupe auquel il est toujours associé : confronter des sources et la validité des contenus $(61,3 \%$, cluster B2), apprendre à communiquer $(61,2 \%$, cluster B2), concevoir et réaliser des productions visuelles, plastiques, sonores ou verbales $(58,7 \%$, cluster A2). Mais elles sont davantage le marqueur d'une représentation du numérique comme outil de communication et de production, plus que de collaboration.

Dans le cluster A1 orienté sur le travail de préparation des séquences de cours avec les collègues, seuls deux pourcentages apparaissent élevés pour le numérique : l'usage de la messagerie électronique $(84,1 \%)$ et celui de l'ordinateur personnel ou professionnel $(68,1$ et $76,4 \%)$.

Concernant la régression PLS, les résultats vont dans le même sens : le numérique y apparaît comme une variable contributive associée au travail en groupe (cf.: V2 «Être persuadé que de nombreuses compétences sont améliorables par le travail en groupe ET par l'usage du numérique ") ou affiche un ICR très modeste : V8 « Usage des supports numériques pour travailler avec leurs collègues " [3,7 à l'école, 1,7 au collège]. Seul le collège présente une catégorie de variables spécifique à l'attitude face au numérique dans ses résultats, mais avec le quatrième ICR sur les six identifiés : C2 «L'attitude sur les technologies du numérique et ses usages " [ICR total $=9,5]$. Ces résultats amènent donc à relativiser fortement l'importance de la place du numérique pour expliquer les pratiques de travail collectif chez les enseignants et l'évolution de la forme scolaire en général (Cerisier, 2016), même s'ils n'excluent pas un usage du numérique ancré dans ces pratiques pour travailler entre pairs et préparer sa classe (comme l'attestent les enquêtes Profetic: ministère de l'Éducation nationale, 2017; 2018). Une interprétation possible est que les outils et ressources numériques habituellement présents dans la classe n'ayant pas été spécifiquement conçus pour soutenir ce type d'activités collectives, ils ne sont pas perçus comme utiles par les enseignants dans ce cadre, à la différence des supports papier ou autres matériels favorables aux manipulations et expérimentations.

\section{Conclusion}

Les principaux résultats de notre enquête offrent une description fine des pratiques déclarées des enseignants, jusqu'à présent peu exposées par la littérature. Les apports sont multiples. Cela permet une proposition de catégorisation des pratiques de travail collectif à partir d'un large échantillon d'enseignants de cycle 3 et 4 sur un territoire donné en France. Les principales catégories identifiées par approche statistique viennent confirmer des travaux antérieurs et proposent des dimensions nouvelles sur les collectifs enseignants ou apprenants perçus comme auto et/ou hétérocentrés. Cette étude permet également l'identification de variables prédictives expliquant la fréquence de leur travail collectif en classe et la relativisation de l'impact des usages du numérique dans ces catégorisation et identification. Elle répond également à un enjeu éducatif identifié par des auteurs comme Dupriez (2010) : le travail collectif des enseignants n'aura une incidence réelle sur leur travail que s'il porte directement sur leurs pratiques professionnelles quotidiennes, c'est-à-dire sur les actions pédagogiques spécifiques qu'ils réalisent au 
sein des classes. Selon lui, l'enjeu est donc de développer un travail collectif faisant fi des discours conventionnels, mais ancré dans le travail quotidien des enseignants, dans les gestes professionnels qu'ils posent, dans les difficultés qu'ils rencontrent face à leurs élèves. D'une certaine manière, notre étude y apporte sa pierre en offrant une description fine de ces pratiques professionnelles du quotidien entre pairs et en classe. Pour autant, et malgré sa diversité de variables, elle n'interroge qu'une partie des dimensions identifiées par les études scientifiques menées sur le travail collectif chez les enseignants (Lessard et al., 2009; Vangrieken et al., 2016), parmi lesquelles figurent également : les préoccupations pédagogiques, le sentiment de compétence (savoir agir ensemble), la personnalité (volonté), le cadre organisationnel (établissement, histoire collective locale) et le fonctionnement des équipes (entité, potentiel, cohésion, comportements, résultats visés).

Pour la suite de notre recherche, les perspectives envisagées sont multiples. En particulier, il semble intéressant d'associer les attributs des répondants pour chaque cluster de pratiques identifié ici afin d'en dégager d'éventuels profils d'enseignants pour chacun (par ancienneté ou par discipline enseignée, par exemple). Cette étude invite aussi à inverser le point de vue sur le numérique (Cerisier, 2020), en constatant ce que le numérique fait à l'école et en expérimentant des interfaces homme-machine de nouvelle génération qui prennent en compte à la fois cette diversité des pratiques collectives existantes et les autres besoins que nous avons pu identifier dans le cadre du projet e-TAC, à savoir :

- la mise en œuvre d'activités diversifiées et la mobilisation de compétences du socle autant dans les dimensions du «pouvoir produire ensemble» que du «pouvoir mettre en commun des connaissances $»$;

- un usage plus proche des supports physiques, notamment papier, très largement préférés aux actuels dispositifs numériques de la classe, en plaidant pour de nouvelles formes interactionnelles plus tangibles;

- des espaces partagés de travail permettant aux élèves de travailler à 3 ou 4, mais aussi aux enseignants de concevoir ensemble leurs activités, que ce soit au sein de leurs classes et établissements ou à l'extérieur.

\section{Liste de références}

Baudrit, A. (2007). La formation des enseignants aux méthodes d'apprentissage coopératif : perspectives internationales. Savoirs, 14, 73-92.

Butori, R., et Parguel, B. (2010). Les biais de réponse-Impact du mode de collecte des données et de l'attractivité de l'enquêteur. Actes du $26^{e}$ Congrès international de l'AFM, Le Mans-Angers, France.

Cerisier, Jean-F. (2016). La forme scolaire à l'épreuve du numérique. In Bonfils, P., Dumas, P. et Massou, L., dirs. Numérique et éducation. Dispositifs, jeux, enjeux, hors jeux. Nancy, PUN-Editions Universitaires de Lorraine, 195210.

Cerisier, J-F. (2020). Faut-il renoncer au numérique pour l'éducation? The Conversation France, 18 juin. Récupéré de : https://theconversation.com/faut-il-renoncer-au-numerique-pour-leducation-140765

Conein, B. (2004), Communautés épistémiques et réseaux cognitifs: coopération et cognition distribuée. Revue d'économie politique, 113, 141-159.

Cristol, D. (2017). Les communautés d'apprentissage : apprendre ensemble. Savoirs, 43(1), 10-55.

Cristol, D. (2016). Peut-on créer des communautés d'apprentissage? Éducation permanente, 207, 155-163.

Dillenbourg, P. (1999). «What do you mean by collaborative learning? ». In Dillenbourg P., dir. Collaborative-learning: Cognitive and Computational Approaches. Oxford : Elsevier, 1-19.

Dupriez, V. (2010). Le travail collectif des enseignants : au-delà du mythe. Travail et formation en éducation, 7 . Récupéré de : $\underline{\text { http://journals.openedition.org/tfe/1492 }}$ 
Gibert, A-F. (2018). Le travail collectif enseignant, entre informel et institué. Dossier de veille de l'IFÉ, 124. Lyon : ENS de Lyon. Récupéré de : http://veille-et-analyses.enslyon.fr/DA/detailsDossier.php?parent=accueil\&dossier=124\&lang=fr

Giraudeau, P., Olry, A., Roo, J. S., Fleck, S., Bertolo, D., Vivian, R. and Hachet, M. (2019). CARDS: A Mixed-Reality System for Collaborative Learning at School. In Proceedings of the 2019 ACM International Conference on Interactive Surfaces and Spaces, 55-64

Jouët, J. (2000). Retour critique sur la sociologie des usages. Réseaux, 100(18), 487-521.

Lê, S., Josse, J. et Husson, F. (2008). FactoMineR: An R Package for Multivariate Analysis. Journal of Statistical Software. 25(1), 1-18.

Lessard, C., Kamanzi, P. et Larochelle, M. (2009). De quelques facteurs facilitant l'intensification de la collaboration au travail parmi les enseignants : le cas des enseignants canadiens. Éducation et sociétés, 23(1), 59-77.

Loup-Escande, E., Dominjon, L., Perret, D., Erhel, S., Jamet, E., Michinov, N., Andriot, C., Gravez, P. and Ragot, M. (2013). La démarche de Conception Centrée-Utilisateur en Réalité Virtuelle: I'exemple du projet VirtualiTeach. In Journées de l'Association Française de Réalité Virtuelle.

Marcel, J., Dupriez, V. et Périsset Bagnoud, D. (2007). Le métier d'enseignant : nouvelles pratiques, nouvelles recherches. In Marcel, J-F., dir. Coordonner, collaborer, coopérer: De nouvelles pratiques enseignantes. Louvain-la-Neuve : De Boeck Supérieur, 7-17.

Maubant, P. (2014). Le travail collectif enseignant : allant de soi, effet de mode convenu ou analyseur décalé de la professionnalité enseignante? Questions Vives, 21. Récupéré de : http://journals.openedition.org/questionsvives/1514

Mevik, B-H., Wehrens, R. et Hovde Liland, K. (2019). PIs : Partial Least Squares and Principal component Regression. R package version 2.7-1. Récupéré de : https://CRAN.R-project.org/package=pls

Ministère de l'Éducation nationale. (2017). Rapport complet PROFETIC (1er degré). Récupéré de : https://eduscol.education.fr/cid60867//-enquete-profetic.html

Ministère de l'Éducation nationale. (2018). Rapport PROFETIC. Récupéré de : https://eduscol.education.fr/cid60867//enquete-profetic.html

Norman, D. A. and Draper, S. W. (1986). User Centered System Design; New Perspectives on Human-Computer Interaction. NJ, USA : L. Erlbaum Assoc. Inc., Hillsdale.

Orellana, I. (2005). L'émergence de la communauté d'apprentissage ou l'acte de recréer des relations dialogiques et dialectiques de transformation du rapport au milieu de vie. In L. Sauvé, L., Orellana, I. et Van Steenberghe, E., dirs. Éducation et environnement. Un croisement de savoirs. Cahiers scientifiques de l'Acfas, 104, 67-84.

Oviatt, S. (2006). Human-centered design meets cognitive load theory: designing interfaces that help people think. In Proceedings of the 14th ACM international conference on Multimedia, 871-880.

Poteaux, N. (2017). Usage des outils numériques : croyances et connaissances des enseignants. In Massou, L. et LavielleGutnik, N., dirs. Enseigner à l'université avec le numérique : Savoirs, ressources, médiations. Louvain-la-Neuve : De Boeck Supérieur, 21-41.

Reuter, Y., Cohen-Azria, C., Daunay, B., Delcambre, I. and Lahanier-Reuter, D. (2010). Dictionnaire des concepts fondamentaux des didactiques. Bruxelles : De Boeck Université.

Tenenhaus, M. (1998). La régression PLS : Théorie et pratique. Paris : Editions TECHNIP.

Tondeur, J., Hermans, R., van Braak, J., et Valcke, M. (2008). Exploring the link between teachers' educational belief profiles and different types of computer use in the classroom. Computers in Human Behavior, 24(6), 2541-2553.

Trudel L., Simard C. et Vonarx N. (2007). La recherche qualitative est-elle nécessairement exploratoire? Recherches qualitatives, hors série, 5, 38-45.

Spillane J., Shirrell M. et Hopkins M. (2016). Designing and deploying a Professional Learning Community (PLC) organizational routine: Bureaucratic and collegial arrangements in tandem. Les dossiers des sciences de l'éducation, 35, 97-122.

Vangrieken, K., Dochy, F., Raes, R. et Kyndt, E. (2015). Teacher collaboration: A systematic review, Educational Research Review, 15, 17-40.

Vangrieken, K., Dochy, F. et Raes, E. (2016). Team learning in teacher teams: team entitativity as a bridge between teams-in-theory and teams-in-practice. European Journal of Psychology of Education, 31.

Vigneau, E., Chen M. et Qannari, E.M. (2015). ClustVarLV: An R Package for the Clustering of Variables around Latent Variables. $R$ Journal, 7(2), 134-148. 